This document is confidential and is proprietary to the American Chemical Society and its authors. Do not copy or disclose without written permission. If you have received this item in error, notify the sender and delete all copies.

\title{
Vibrational Relaxation and Redistribution Dynamics in Ru(II) Polypyridyl-Based Charge-Transfer Excited States: A Combined Ultrafast Electronic and Infrared Absorption Study
}

\begin{tabular}{|r|l|}
\hline Journal: & The Journal of Physical Chemistry \\
\hline Manuscript ID & jp-2018-061974.R1 \\
\hline Manuscript Type: & Article \\
\hline Date Submitted by the Author: & n/a \\
\hline Complete List of Authors: & $\begin{array}{l}\text { Brown, Allison; Michigan State University, Department of Chemistry } \\
\text { McCusker, Catherine; East Tennessee State University, Chemistry } \\
\text { Carey, Monica; Michigan State University, Department of Chemistry } \\
\text { Blanco-Rodriguez, Ana; Queen Mary University of London, School of } \\
\text { Biological and Chemical Sciences } \\
\text { Towrie, Michael; Rutherford Appleton Laboratory, Research Complex at } \\
\text { Harwell } \\
\text { Clark, Ian; Science \& Technology Facilities Council, Central Laser Facility } \\
\text { Vlcek, Antonin; Queen Mary, University of London, SBCS } \\
\text { McCusker, James; Michigan State University, Department of Chemistry }\end{array}$ \\
\hline
\end{tabular}


Vibrational Relaxation and Redistribution Dynamics in

Ru(II) Polypyridyl-based Charge-Transfer Excited States:

A Combined Ultrafast Electronic and Infrared Absorption Study

\begin{abstract}
.
Ultrafast time-resolved electronic and infrared absorption measurements have been carried out on a series of $\mathrm{Ru}(\mathrm{II})$ polypyridyl complexes in an effort to delineate the dynamics of vibrational relaxation in this class of charge transfer chromophores. Time-dependent density functional theory calculations performed on compounds of the form $\left[\mathrm{Ru}(\mathrm{CN}-\mathrm{Me}-\mathrm{bpy})_{\mathrm{x}}(\mathrm{bpy})_{3-\mathrm{x}}\right]^{2+}(\mathrm{x}=1-3$ for compounds $\mathbf{1}-\mathbf{3}$, respectively, where CN-Me-bpy is 4,4'-dicyano-5,5'-dimethyl-2,2'-bipyridine and bpy is 2,2'-bipyridine) reveal features in their charge-transfer absorption envelopes that allow for selective excitation of the $\mathrm{Ru}(\mathrm{II})-(\mathrm{CN}-\mathrm{Me}-\mathrm{bpy})$ moiety, the lowest-energy MLCT state(s) in each compound of the series. Changes in band shape and amplitude of the time-resolved differential electronic absorption data are ascribed to vibrational cooling in the CN-Me-bpy-localized ${ }^{3}$ MLCT state with a time constant of $8 \pm 3$ ps in all three compounds. This conclusion was corroborated by picosecond time-resolved infrared absorption
\end{abstract}


measurements; sharpening of the $\mathrm{CN}$ stretch in the ${ }^{3} \mathrm{MLCT}$ excited state was observed with a time constant of $3.0 \pm 1.5 \mathrm{ps}$ in all three members of the series. Electronic absorption data acquired at higher temporal resolution revealed spectral modulation over the first 2 ps occurring with a time constant of $\tau=$ $170 \pm 50$ fs, in compound 1; corresponding effects are significantly attenuated in compound $\mathbf{2}$ and virtually absent in compound $\mathbf{3}$. We assign this feature to intramolecular vibrational redistribution (IVR) within the ${ }^{3} \mathrm{MLCT}$ state and represents a rare example of this process being identified from time-resolved electronic absorption data for this important class of chromophores. 


\section{Introduction}

A number of photophysical and photochemical conversions occur on ultrafast timescales, with phenomena such as intersystem crossing, internal conversion, and even photo-induced bond cleavage reactions being reported with rate constants in excess of $10^{12} \mathrm{~s}^{-1}$ in a wide variety of chemical systems. ${ }^{1-5}$ The occurrence of these processes on time scales comparable to the vibrational oscillations of a molecule raises the question as to whether they might be initiated from so-called "hot" states, i.e., vibrational levels of the electronic excited state other than that corresponding to $v=0$. Such a circumstance would imply competition between the electronic and structural features of photoexcited molecules and therefore represent an important aspect of excited-state evolution in molecular systems. While it is necessary to understand this connection with regard to fundamental aspects of photophysics and photochemistry, the details of the interplay between electronic and structural degrees of freedom under the influence of an optical bias have more significant implications for the design and use of chromophore assemblies in applications ranging from optoelectronics to solar energy conversion.

As a class, the excited-state dynamics of transition metal complexes are dominated by the sorts of non-radiative processes just described; even in charge-transfer complexes, all but the most highly emissive compounds dissipate the majority of the photo-induced excess energy via non-emissive pathways. Non-radiative decay along one electronic potential energy surface in large molecules has two major components, namely intermolecular vibrational relaxation (herein referred to as vibrational cooling), and intramolecular vibrational energy redistribution (IVR). Vibrational cooling is the transfer of vibrational energy from the excited molecule into its surroundings (e.g., solvent), resulting in a net loss of energy within the chromophore: this corresponds to thermalization of the excited state. Intramolecular vibrational energy redistribution, on the other hand, is the transfer of vibrational energy from an excited vibrational mode(s) of the chromophore into other vibrational modes that were not directly coupled to the electronic excitation. In this process there is no net loss of energy from the chromophore; rather, the absorbed energy is redistributed to the various vibrational degrees of freedom of the molecule. To the extent that IVR affects the spatial localization of the excitation energy, this process can lead to differences in excited-state reactivity based on the geometric and compositional properties of the system. ${ }^{1,6-13}$ Despite its important role in excited state dynamics, detailed investigations of vibrational relaxation dynamics in transition metal-based compounds are relatively limited in number. ${ }^{3,6-9,12,14-21}$

In considering the general problem of understanding the mechanism of excited-state evolution in transition metal complexes, it occurred to us that an examination of this issue must confront two critical problems. First, the use of transient electronic absorption spectra to infer processes related to vibrational dynamics in transition metal-containing systems is a largely untested paradigm. In our work, for example, 
assignments of kinetics being due to vibrational relaxation have been arrived at through a process of elimination: once electronic structure changes were deemed to be complete, small amplitude and/or band shape modulations in the differential absorption spectra occurring on longer time scales (but orders of magnitude faster than ground-state recovery) were ascribed to vibrational cooling., ${ }^{2,22-24}$ One can make a reasonable argument based on anticipated changes in Franck-Condon factors in support of this assertion, but it is still an indirect means of probing vibrational dynamics in electronic excited states. The development of ultrafast vibrational spectroscopies over the past several years presents tremendous opportunities for the direct measurement of vibrational dynamics in charge-transfer excited states, ${ }^{6-9,12-}$ $16,20,21$ but these methodologies tend to be more technically challenging and therefore not as commonly employed in the physical-inorganic community as electronic absorption spectroscopy.

The second issue relates to the density of vibrational states one often encounters in transition metal complexes. The benchmark studies of metal carbonyl complexes by Harris, ${ }^{25}$ Simon, ${ }^{26,27}$ and others, ${ }^{8}$ as well as the late Paul Barbara's work on cyano-bridged mixed-valence ruthenium and iron dimers ${ }^{28}$ were successful in part because the $\mathrm{C}-\mathrm{O}$ and $\mathrm{C}-\mathrm{N}$ stretches of these moieties occur in isolated regions of the infrared spectrum. Compounds such as $\left[\mathrm{Ru}(\mathrm{bpy})_{3}\right]^{2+}$ present a far greater challenge for probing vibrational dynamics due to the large number of vibrational degrees of freedom congested in a relatively narrow spectral window; even excluding the protons on each bpy ligand, the seven vibronically active modes associated with the ${ }^{1} \mathrm{~A}_{1} \rightarrow{ }^{1} \mathrm{MLCT}$ absorption ${ }^{29}$ can theoretically couple to a bath comprised of nearly 100 normal modes. In this context, the studies of Mathies ${ }^{30}$ and Browne and McGarvey ${ }^{31}$ in particular represent important benchmarks along these lines.

To address these challenges, we sought to develop a molecular platform that would be amenable to study through the use of both ultrafast electronic and vibrational spectroscopies while at the same time allow for unambiguous identification and differentiation of vibrational relaxation and IVR. The series of compounds we have prepared to achieve this goal is illustrated in Chart 1. Following the example of Barbara, we have chosen the $\mathrm{CN}$ moiety as our probe for vibrational dynamics due to its spectral isolation; by placing this group at the 4 and 4' positions of the bipyridine ligand, conjugation into the $\pi$ system of the rings couples it to the MLCT manifold of the chromophore and thus allows it to act as a reporter for excited-state charge-transfer dynamics. We have previously described the synthesis, electrochemistry, as well as steady-state and nanosecond time-resolved absorption, emission, and infrared spectroscopic properties of this series of compounds: ${ }^{32}$ with this report, we shift our attention to their ultrafast excited-state dynamics. Results from time-dependent density functional theory calculations will be described that lend support to our previous assertion ${ }^{32}$ that the broad charge-transfer envelopes exhibited in the visible absorption spectra of these compounds can be viewed as a superposition of 
absorption features associated with the $\mathrm{CN}$ - and un-substituted ligand(s) in the low and high energy regions, respectively. Femtosecond time-resolved electronic absorption and picosecond infrared absorption data were then acquired on all members of the $\left[\mathrm{Ru}(\mathrm{CN}-\mathrm{Me}-\mathrm{bpy})_{\mathrm{x}}(\mathrm{bpy})_{3-\mathrm{x}}\right]^{2+}$ series $(\mathrm{x}=1-3$ for compounds $\mathbf{1}-\mathbf{3}$, respectively) in order to assess the extent to which the former can be used to draw conclusions concerning the dynamics of vibrational relaxation. Finally, data will be presented in the form of sub-100 fs time-resolved electronic absorption spectra that provide novel insights into the time scale of IVR in this class of charge-transfer chromophores.

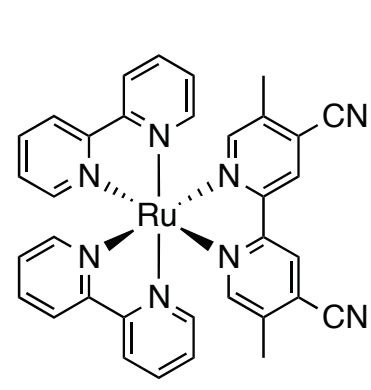

1

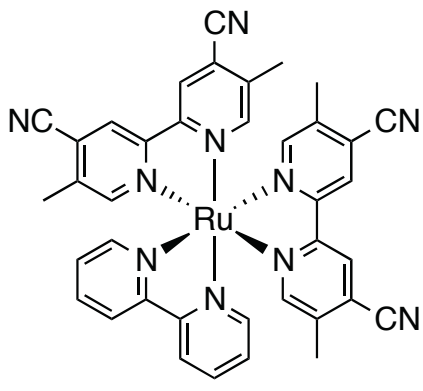

2

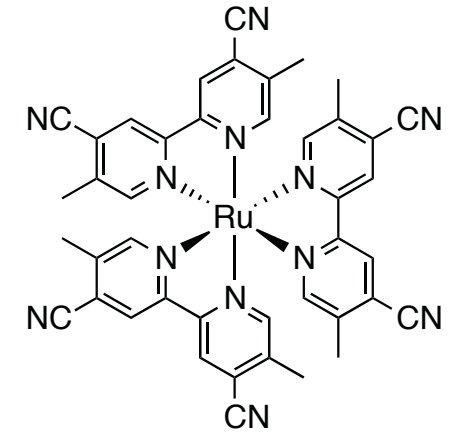

3

Chart 1. Drawings of the cations of $\left[R u(b p y)_{2}(C N-M e-b p y)\right]\left(P F_{6}\right)_{2}(\mathbf{1}),\left[R u(b p y)(C N-M e-b p y)_{2}\right]\left(P F_{6}\right)_{2}$ (2), and $\left[R u(C N-M e-b p y)_{3}\right]\left(P F_{6}\right)_{2}(3)$.

\section{Experimental}

2.1. Synthesis. All members of the $\left[\mathrm{Ru}(\mathrm{CN}-\mathrm{Me}-\mathrm{bpy})_{\mathrm{x}}(\mathrm{bpy})_{3-\mathrm{x}}\right]^{2+}$ series (where $\mathrm{x}=1-3$ for compounds $\mathbf{1}-\mathbf{3}$ ), respectively) were prepared as described previously. ${ }^{32}$

2.2. Time-Resolved Electronic Absorption Spectroscopy. Femtosecond time-resolved electronic absorption data were acquired using a spectrometer designed to emulate a previously reported system. ${ }^{33} \mathrm{~A}$ commercial Ti:Sapphire oscillator (Coherent: Mantis) provides $500 \mathrm{~mW}$ at $80 \mathrm{MHz}$ as the seed for a Nd:YLF pumped Ti:Sapphire regenerative amplifier (Coherent: Legend Elite USP), producing a $1 \mathrm{kHz}$ train of $\sim 40$ fs pulses $(\sim 1.3 \mathrm{~mJ})$ at $800 \mathrm{~nm}$. The output is split 70/30 with the larger portion being used to pump an optical parametric amplifier (Coherent: OPerA Solo), generating a tunable pump source. The smaller portion is used to pump a second OPA (Coherent: OperA Solo) for single-wavelength measurements or, for full spectrum data collection, attenuated to generate a white light continuum probe in the single filament regime by focusing $\sim 2 \mu \mathrm{J}$ into a $6 \mathrm{~mm}$-thick $\mathrm{CaF}_{2}$ window attached to a continually moving transducer. The pump OPA output is passed through an optical chopper (446 Hz), directed on a computer controlled translation stage (Aerotech: $3.3 \mathrm{fs} /$ step time resolution), and softly focused into a 1 
$\mathrm{mm}$ path length quartz cell containing a solution of the sample. The pump polarization is horizontal with excitation energy in the range of 3-6 $\mu \mathrm{J}$ at the sample; the power dependence of all data acquired was checked to verify linear response. The probe is polarized at $54.7^{\circ}$ with respect to the pump to minimize anisotropic contributions to the observed kinetics. Off-axis parabolic mirrors are used to collimate and focus the white light into the sample at an acute angle $\left(\sim 5^{\circ}\right)$ with respect to the pump. Cross-correlation data acquired in neat solvent were used to determine time zero as well as the instrument response function at various pump-probe combinations. The latter were fit to Gaussian curves with a full width at half maximum of $75 \pm 10 \mathrm{fs}$.

Single wavelength kinetics measurements are made by passing the probe through a $10 \mathrm{~nm}$ band-pass filter of the appropriate wavelength positioned after the sample and focused onto an amplified Si photodiode (Thor labs); a reference beam of $800 \mathrm{~nm}$ light (not chopped) is directed onto a similar amplified photodiode. The signal and reference are coupled to a digital lock-in amplifier (Stanford Research: SR810) synchronized to the optical chopper. The reference beam intensity is attenuated to match that of the signal beam in order to account for laser power fluctuations. Single wavelength plots of kinetic data represent an average of 6-12 scans where a single scan corresponds to data collected at each of the time points visited by the translation stage in a forward direction averaged with data collected at the same time points in the reverse direction.

Full spectrum data were acquired by coupling the white light probe into a spectrograph (Spex 270M) and dispersed horizontally onto a photodiode array (Hamamatsu HC233-0900 with a NMOS C9564 array). The grating angle can be changed with respect to the incoming light in order to select the desired center wavelength; spectra spanning $\sim 300 \mathrm{~nm}$ are collected as a function of pump stage. Dark counts are collected in the absence of the probe beam at the beginning of each experiment to identify and account for laser scatter, whereas spectra collected at the beginning of each scan at negative time are used as the reference. The data reported herein are typically an average of 24-32 scans. Chirp correction due to dispersion of the probe beam was accomplished by acquiring traces of neat solvent capturing the crosscorrelation signal at each wavelength; the "time zero" for each wavelength was taken to be the median time of the response. A plot of the wavelength vs. corresponding "time zero" was then fitted to the builtin double exponential with an x-offset function available in the Igor Pro software suite (v. 6.2.2). This fit was applied to the full data set, effectively correcting the timing for each full spectrum. The solvent response (which takes the form of stimulated Raman scattering as well as cross-phase modulation) can be seen during the first $\sim 100$ fs: these spectral traces were therefore not considered in the data analysis. Further details concerning the application of singular value decomposition and global analysis to differentiate genuine spectral features from artifacts can be found in the Supplementary Materials. 
Spectrophotometric grade acetonitrile (Aldrich Chemical Co.), which had been freeze-pump-thaw degassed prior to use, was used as the solvent. Samples were prepared with an absorbance of $0.4-0.8$ at the excitation wavelength in air-free $1 \mathrm{~mm}$ pathlength cells. Ground state absorption spectra were taken before and after data collection in order to assess sample integrity. Compounds $\mathbf{1}$ and $\mathbf{2}$ showed no changes in their UV-visible absorption spectra and no other signs of decomposition over several days. Compound $\mathbf{3}$ showed a small change in the absorption spectrum over a two-day period, so a fresh sample of $\mathbf{3}$ was prepared for each measurement.

2.3. Time-Resolved Infrared Spectroscopy. Time-resolved infrared absorption (TRIR) data were collected at the Rutherford Appleton Laboratory using an instrument that has been described previously. ${ }^{33}$ Samples were dissolved in nitromethane and placed in $0.1 \mathrm{~mm}$ pathlength cells with absorbances of $\sim 0.7$ at the excitation wavelength, resulting in ground state infrared absorptions in the range of $0.005-0.01$ for the $\mathrm{CN}$ vibration(s). The samples were excited at $490 \mathrm{~nm}$ for compounds 1 and 2 and $475 \mathrm{~nm}$ for compound 3. Unless otherwise stated, all time-resolved IR measurements were made at magic angle. The polarization anisotropy measurements were made with the infrared probe set to horizontal polarization, with the pump (both $400 \mathrm{~nm}$ and $490 \mathrm{~nm}$ excitation experiments) varied from horizontal to vertical using half waveplates. The raw data was baseline corrected with a spline function in Igor Pro. The energy axis was calibrated using Equations 1 and 2,

$$
\begin{gathered}
d=\frac{\frac{1}{\bar{v}_{a}}-\frac{1}{\bar{v}_{b}}}{b-a} \\
\bar{v}_{i}=\frac{1}{\left.\lambda_{a}+d(a-i)\right]}
\end{gathered}
$$

where $\mathrm{d}$ is dispersion, $\mathrm{a}$ and $\mathrm{b}$ are pixels, $\overline{v_{\mathrm{a}}}$ and $\overline{v_{\mathrm{b}}}$ are the energies $\left(\right.$ in $\mathrm{cm}^{-1}$ ) of pixels a and $\mathrm{b}, \overline{v_{\mathrm{i}}}$ is the energy (in $\mathrm{cm}^{-1}$ ) of pixel $\mathrm{i}$, and $\lambda_{\mathrm{a}}$ is the wavelength of pixel a (in $\mathrm{cm}$ ). Dispersion was calculated using the maximum of the excited state absorption and the minimum of the ground state bleach as pixels $a$ and $b$ and using the energies observed in the nanosecond time resolved infrared experiment for $\overline{v_{\mathrm{a}}}$ and $\overline{v_{\mathrm{b}}}$. 32

2.4 Density Functional Theory (DFT) Calculations. Calculations on complexes $\mathbf{1}$ - $\mathbf{3}$ were performed using the Gaussian 03 software package. ${ }^{34}$ Geometry optimizations were carried on the ground states of the compounds using a spin-unrestricted formalism at the B3LYP/LANL2DZ level of theory. ${ }^{35,36}$ No symmetry restrictions were placed on the geometry optimizations. The influence of the solvent environment was accounted for through use of the polarizable continuum model (PCM). ${ }^{37}$ Frequency calculations were performed on the optimized structures to ensure that these geometries corresponded to 
global minima: no imaginary frequencies were obtained for any of the optimized geometries. The first 80 electronic transitions (singlets and triplets) of the optimized structures were calculated by time-dependent DFT using a spin-unrestricted formalism at the B3LYP/LANL2DZ level of theory. The orbitals contributing to each transition were visualized using GaussView.

\section{Results and Discussion}

We have previously reported on the ground state and long-lived excited state properties of complexes $1-3{ }^{32}$ Briefly, electrochemical data in combination with steady-state and nanosecond time-resolved emission, electronic and step-scan infrared absorption measurements were acquired on all members of this series. The electrochemistry revealed that 4,4'-dicyano-5,5'-dimethyl-2,2'-bipyrdine (CN-Me-bpy) is more easily reduced than 2,2'-bipyridine and should therefore house the lowest-energy, thermalized ${ }^{3}$ MLCT excited state in all members of the series depicted in Chart 1 . This was verified by the nearly identical nanosecond differential electronic absorption spectra of $\mathbf{1}-\mathbf{3}$, particularly in the near-UV region which is diagnostic for bipyridyl radical anions; in the specific cases of compounds $\mathbf{1}-\mathbf{3}$, the absorption corresponding to the (CN-Me-bpy) moiety is observed at $\sim 395 \mathrm{~nm}$, a red-shift of ca. $25 \mathrm{~nm}$ from the $\sim 370 \mathrm{~nm}$ feature observed in the excited-state differential spectrum of $\left[\mathrm{Ru}(\mathrm{bpy})_{3}\right]^{2+}$. 14,38 Nanosecond stepscan IR data showed identical red-shifts of $\sim 40 \mathrm{~cm}^{-1}$ for the $\mathrm{CN}$ stretching frequency in compounds $\mathbf{1}-\mathbf{3}$, a clear indication of coupling of the $\mathrm{CN}$ group into the ligand $\pi$ system as well as establishing that the long-lived ${ }^{3}$ MLCT state is localized on a single CN-Me-bpy ligand in all three complexes. This information provides us with the necessary foundation to begin examining the process(es) by which the long-lived, thermalized ${ }^{3} \mathrm{MLCT}$ states of these compounds are formed.

3.1. DFT and TD-DFT Calculations. An understanding of the temporal evolution of the chromophore immediately following photoexcitation requires knowledge about the nature of the initially formed Franck-Condon excited state. The ground-state electronic absorption spectra of compounds $\mathbf{1}-\mathbf{3}$ are plotted in Figure 1. While excitation of compound 3 anywhere within the charge-transfer envelope will place the Franck-Condon state on a CN-Me-bpy ligand, the significant difference in reduction potential for CN-Me-bpy versus bpy implies that the spatial localization of the initial state in compounds $\mathbf{1}$ and $\mathbf{2}$ is likely to be excitation-wavelength dependent. In our previous report, ${ }^{32}$ we provided a qualitative assessment as to which region of the spectrum reflects coupling to which ligand, but more concrete information along these lines was desirable in advance of ultrafast spectroscopic measurements. Resonance Raman spectroscopy is the experimental method of choice in this regard given its inherent selectivity for modes that are vibronically coupled to the selected electronic absorption band; ${ }^{39,40}$ this approach would be particularly useful in the case of compounds $\mathbf{1}$ - $\mathbf{3}$ due to the distinctive nature of the 
$\mathrm{C}-\mathrm{N}$ stretch of the CN-Me-bpy ligand. Unfortunately, our efforts along these lines were unsuccessful due to interference from emission arising from the ${ }^{3} \mathrm{MLCT}$ excited states of these compounds. ${ }^{32} \mathrm{We}$ therefore turned to theory in order to gain further insight into the origin(s) of the transitions making up the chargetransfer features in this system.

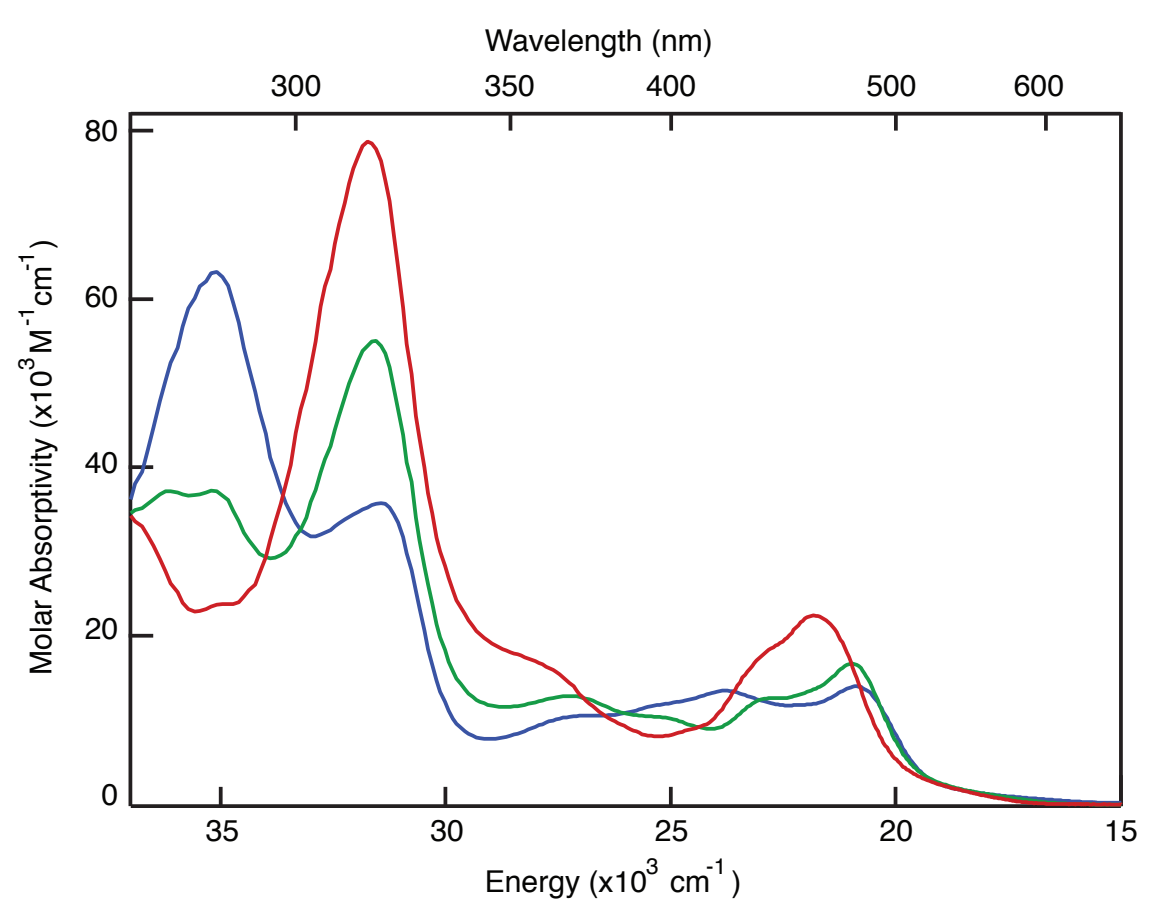

Figure 1. Electronic absorption spectra of $\left[R u(b p y)_{2}(C N-M e-b p y)\right]\left(P F_{6}\right)_{2}(1$, blue line $),[R u(b p y)(C N-$ Me-bpy $\left.)_{2}\right]\left(P F_{6}\right)_{2}\left(2\right.$, green line), and $\left[R u(C N-M e-b p y)_{3}\right]\left(P F_{6}\right)_{2}(3$, red line). The spectra were acquired in $\mathrm{CH}_{3} \mathrm{CN}$ solution.

The ground state geometries for compounds $\mathbf{1}$ - $\mathbf{3}$ were first optimized using a PCM-based dielectric appropriate for $\mathrm{CH}_{3} \mathrm{CN}$, the solvent in which the ground-state and time-resolved excited-state electronic absorption data were acquired: ${ }^{37}$ these optimized geometries were then used as the starting points for time-dependent (TD-DFT) calculations. For each compound, the first 80 transitions (both singlet-singlet and singlet-triplet) were calculated. Figure 2 shows the results for complex 1 along with the dominant orbital contributions to selected absorptive features of the molecule. The blue squares represent the calculated singlet-singlet transitions, whereas the red triangles are the calculated singlet-triplet transitions. The latter were calculated having zero oscillator strength (i.e., $f=0$ ) since spin-orbit coupling was neglected, however, they can still contribute to the experimental spectra. ${ }^{41}$ Finally, the black line corresponds to the experimentally observed electronic absorption spectrum. 
In general, we find excellent overall agreement between the calculated and experimental absorption features for compound 1. In particular, we note how well the calculated transition energies align with the dominant bands in the spectrum without the need for any corrections that are sometimes invoked in TDDFT analyses. ${ }^{35}$ The lowest energy feature near $480 \mathrm{~nm}$ is best described as a metal-to-ligand charge transfer band; the orbital contributions reveal that the transition is from a (mostly) $t_{2 \mathrm{~g}}$-type $\mathrm{d}$ orbital on the ruthenium center to an empty $\pi^{*}$ orbital localized on the CN-Me-bpy ligand with no significant involvement of orbitals associated with the unsubstituted bpy ligands. This is in agreement with expectations based on the electrochemical properties of this compound indicating that the CN-Me-bpy ligand is most easily reduced and should therefore represent the lowest-energy MLCT state(s). ${ }^{32}$ The spectrum becomes more complex at higher energies due to the increasing number of multielectronic configurations that have comparable energies, however, the most prominent features in the range of $400-$ $425 \mathrm{~nm}$ are clearly charge-transfer bands associated with the unsubstituted bpy ligands. The example 
highlighted in Figure 2 has two major contributions of roughly equal weight, one that is best described as a $\mathrm{t}_{2 \mathrm{~g}}$-to-ligand $\pi^{*}$ orbital transition and the other having some added contributions from the CN-Me-bpy ligand in both the ground and excited states. The wavefunctions describing the excited states in these transitions appear to be distributed across the two unsubstituted bpy ligands. Finally, the band centered near $325 \mathrm{~nm}$ is a ligand-localized, $\pi \rightarrow \pi^{*}$ absorption of the CN-Me-bpy ligand.

Analogous calculations carried out on compound $\mathbf{2}$ yielded similar results, i.e., the lowest-energy absorptions being due to MLCT transitions involving the CN-Me-bpy ligands (Figure S1). The higher energy portion of the charge-transfer envelope is slightly more complicated than what was seen for compound 1 insofar as features near $400 \mathrm{~nm}$ derive from charge transfer transitions to both the lone bpy as well as the CN-Me-bpy ligands (although the former represents the dominant contribution); as with compound $\mathbf{1}$, absorptions in the ultraviolet are predominantly ligand-localized, $\pi \rightarrow \pi^{*}$ transitions. In complex 3, the homoleptic nature of the compound means that there is only one possible ligand-type available to house both the initial and thermalized excited state. This is clearly evident from the calculations, which reveal excited states within the charge-transfer manifold involving either one or two of the CN-Me-bpy ligands (Figure S2).

The TD-DFT calculations just described support our previous assessment, ${ }^{32}$ namely that excitation in the low-energy portion of the MLCT absorption envelope in all three complexes in the series will result in a Franck-Condon state that is localized on the CN-Me-bpy ligand, whereas high-energy excitation in compounds 1 and $\mathbf{2}$ places the initial excited state on the unsubstituted, higher energy bpy ligand, thus giving rise to spatially distinct Franck-Condon and lowest-energy, thermalized excited states. Excitedstate evolution in this latter scenario necessarily involves a more complex sequence of events, including interligand electron transfer. In the present study, we will primarily concern ourselves with low-energy excitation where both excited-state formation and thermalization are associated with the same metalligand fragment. This simplifies the problem considerably by limiting the dynamics to energy dissipation in a spatially confined region of the chromophore and should allow us to draw more definitive conclusions concerning the process(es) associated with excited-state thermalization. The details of excited-state evolution following higher energy excitation - which will presumably include the relaxation processes described herein, as well as additional dynamics associated with spatial redistribution of the excited state - will be the subject of a future report.

3.2. Ultrafast Time-Resolved Spectroscopy. Information about excited-state thermalization is often inferred from differential electronic absorption spectra where subtle modulations in band shape (e.g., narrowing of an absorption feature over time) are attributed to vibrational cooling. ${ }^{23}$ Such assignments are reasonable in the absence of significant changes in the overall spectral profile that might suggest a change 
in electronic state, but they are nevertheless still based on indirect evidence. ${ }^{1,10}$ Far preferable is the use of ultrafast infrared and/or Raman spectroscopy where vibrational transitions are probed directly. With these techniques, vibrational relaxation is generally manifested as a narrowing and blue shifting of the infrared spectrum. ${ }^{9,20,21,29,42-45}$ The former occurs because the Franck-Condon excitation creates a distribution of vibrationally excited states which subsequently relax to the $v=0$ vibrational state, causing the spectrum to narrow, whereas the anharmonic nature of the potential surface gives rise to the concomitant blueshift. $^{6}$

Electronic absorption and vibrational spectroscopies together can offer complementary and thus more complete information about excited state dynamics. Maçôas and coworkers, for example, studied $\mathrm{Cr}(\mathrm{acac})_{3}$ by ultrafast infrared absorption spectroscopy. ${ }^{46}$ Biexponential ground state recovery kinetics associated with the ligand $\mathrm{C}=\mathrm{C}$ and $\mathrm{C}=\mathrm{O}$ stretches were observed, in contrast to the single exponential kinetics documented from the time-resolved electronic absorption measurements reported by Juban and McCusker. ${ }^{23}$ Combining these results allowed for the construction of a more sophisticated model than was possible from either study alone. The system was observed to bifurcate at or near the Franck-Condon region to produce a sub-100 fs ISC to the ${ }^{2} \mathrm{E}$ state in competition with back-ISC to the ${ }^{4} \mathrm{~T}_{2}$ state, the latter leading to a $15 \mathrm{ps}$ internal conversion back to the ground state. ${ }^{46}$ With this example in mind, we carried out both electronic and infrared time-resolved absorption measurements on complexes $\mathbf{1}-\mathbf{3}$ in an effort to create a well-defined benchmark for exited-state evolution in the $\mathrm{Ru}(\mathrm{II})$ polypyridyl class of chromophores.

3.2.1. Time-Resolved Electronic Absorption Measurements. A useful approach to the study of the excited-state evolution of transition metal complexes is to essentially work backwards in time, that is, identify when the spectroscopic features characteristic of the lowest-energy excited state are established, then analyze the evolution to that point in order to determine the nature of the ultrafast dynamics involved in its formation. Figure 3 compares the differential electronic absorption spectrum of the fully relaxed ${ }^{3}$ MLCT excited state for compound 1 with one acquired at a delay of 15 ps following excitation at 480 nm. ${ }^{32}$ Three main features are apparent: (1) the aforementioned absorption in the near-UV at $\sim 395 \mathrm{~nm}$, which is associated with the CN-Me-bpy-based radical anion, (2) a strong bleach in the mid-visible due to loss of the ground-state charge-transfer band, and (3) a weak, broad feature extending to the red past 500 $\mathrm{nm}$ previously assigned to a superposition of absorptions of the CN-Me-bpy ${ }^{-}$species and an LMCT band associated with the $\mathrm{Ru}^{\mathrm{III}}$-bpy chromophore present in the excited state. ${ }^{22,32}$ Superimposed on these data is a differential spectrum corresponding to a time delay of $\Delta t=15 \mathrm{ps}$. It can be seen that the two spectra closely match one another, a clear indication that the ${ }^{3} \mathrm{MLCT}$ is fully formed by $15 \mathrm{ps}$. This is wholly consistent with the body of work that has been published on compounds of this class but in the present 


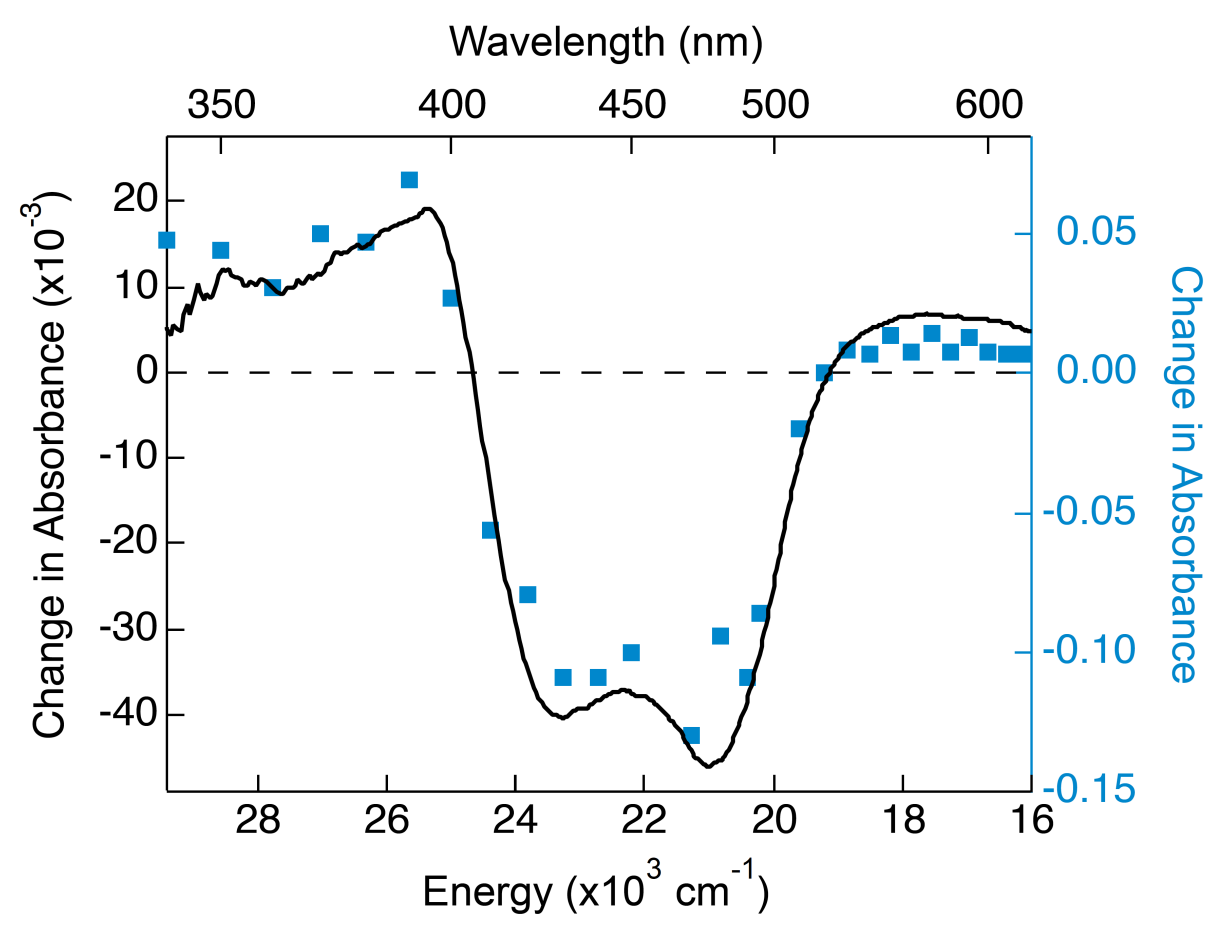

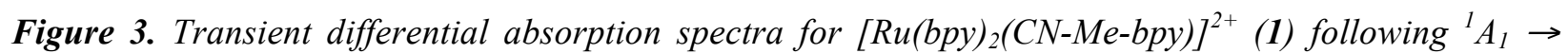
${ }^{I}$ MLCT excitation at $490 \mathrm{~nm}$. The black line corresponds to the differential spectrum at a time delay of $\Delta t$ $=15$ ps, whereas the blue squares represent amplitudes derived from single-exponential fits of the kinetics of ground-state recovery acquired at each wavelength from nanosecond time-resolved absorption measurements.

context serves as an important baseline for the interpretation of spectra acquired at earlier time delays.

In Figure 4 are plotted differential electronic absorption spectra for complex 1 over the first 25 ps following excitation at $490 \mathrm{~nm}$. Qualitatively, it can be seen that the main spectroscopic features described above that are characteristic of a CN-Me-bpy-based MLCT excited state are established by ca. 1 ps. ${ }^{47}$ Furthermore, there are no substantial changes in the overall spectral profile within this time window; of particular note is the fact that there are no significant perturbations to the isosbestic points in the excited-state/ground-state differential spectra within this time window, consistent with the notion that two and only two electronic states are being sampled (i.e., the ground and the lowest-energy ${ }^{3} \mathrm{MLCT}$ states). These observations, coupled with the fact that excitation at $490 \mathrm{~nm}$ places the Franck-Condon state spatially coincident with the lowest-energy excited state of the compound, allows us to conclude that the electronic structure evolution of complex 1 is complete within 1 ps following excitation at $490 \mathrm{~nm}$. 
(a)

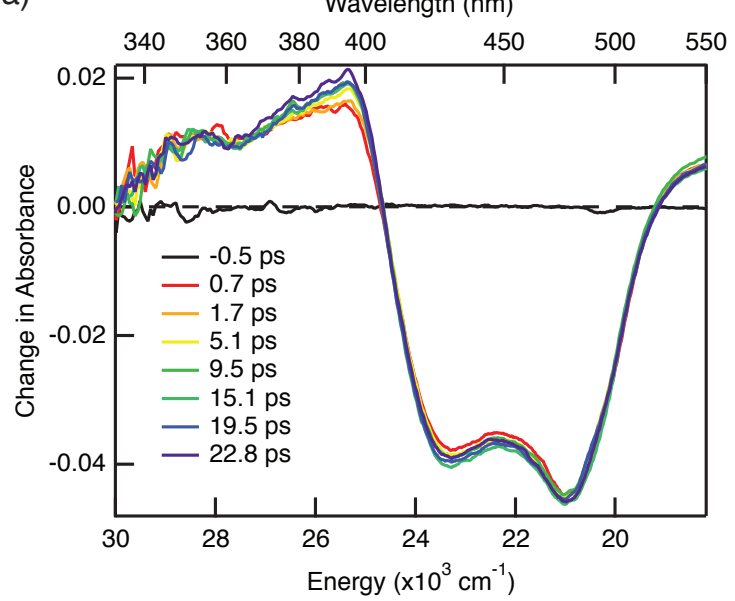

(b)

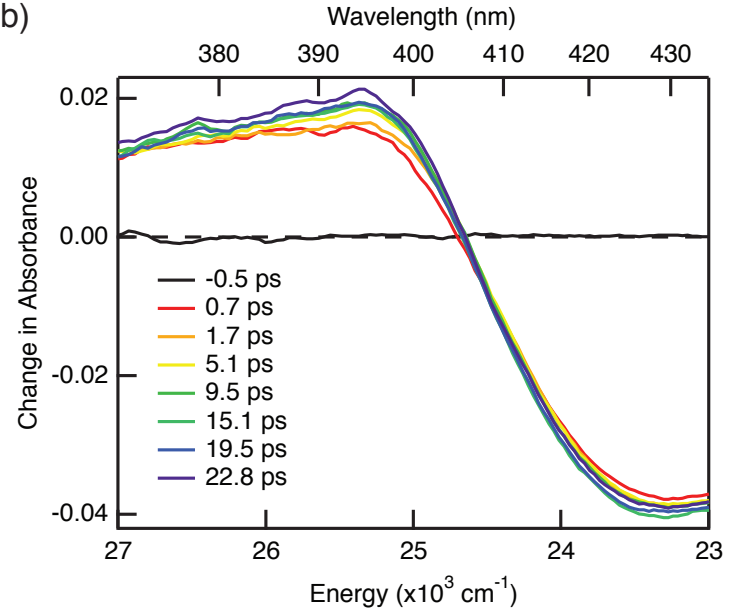

Figure 4. (a) Differential absorption spectra acquired for $\left[\mathrm{Ru}(\mathrm{bpy})_{2}(\mathrm{CN}-\mathrm{Me} \text {-bpy) }]^{2+}\right.$ (1) in $\mathrm{CH}_{3} \mathrm{CN}$ solution following ${ }^{I} A_{1} \rightarrow{ }^{I} M L C T$ excitation at $490 \mathrm{~nm}$. Time delays for each spectrum are indicated in the inset. (b) An expanded view of the ultraviolet and near-UV region of the spectra plotted in part (a).

Although the overall spectral profile is largely invariant, a closer examination reveals some subtle changes occurring over the temporal window illustrated in Figure 4. This is most clearly evident in Figure $4 \mathrm{~b}$, which is an expanded view of the blue region of the spectrum. When examining these spectra, it is important to keep in mind that they reflect a superposition of absorptions arising from the excited state in this case the ${ }^{3}$ MLCT state of complex $\mathbf{1}$ - and the loss of the ground state absorption. Since the latter is instantaneous and static, any modulations in the differential spectra are solely due to processes associated with the excited state. We see the most significant changes blue of the isosbestic point at $405 \mathrm{~nm}$ where the ligand radical anion present in the ${ }^{3} \mathrm{MLCT}$ state dominates the spectrum. ${ }^{48}$ The signal appears to exhibit an overall increase in intensity over the course of $\sim 20 \mathrm{ps} ;{ }^{42,49,50}$ the peak, which is not very prominent at early times (i.e., the absorption profile in the range of $370-395 \mathrm{~nm}$ is fairly flat), becomes better defined as the system evolves in time. If we then look at the spectrum in the bleach region near 425 $\mathrm{nm}$, we see a similar albeit less pronounced trend over the same time window but in the opposite sense: the signal is becoming more negative while shifting slightly toward the blue over time. These changes can be explained quite easily in terms of the sharpening of an excited-state absorption feature whose differential absorption maximum is blue of $410 \mathrm{~nm}$. This is precisely the type of signature expected for vibrational cooling as the system dissipates energy and the population distribution across the various vibrational modes of the molecule shifts toward lower-energy vibrational quanta. These lower-energy vibrational states are characterized by wavefunctions whose amplitudes are more centered about the 
equilibrium geometry of the excited state; as a result, the Franck-Condon factors that serve to define the breadth of the absorption feature become distributed over a much narrower range of nuclear displacements, resulting in a sharpening of the absorption band. Concomitant with this sharpening, the contribution of the excited-state electronic absorption toward offsetting the loss of ground-state absorption on the red side of the isosbestic is diminished, thereby increasing the magnitude of the bleach signal. In the case of the differential spectra shown in Figure 4, the manifestation of this process is attenuated due to superposition of these absorptive features on the ground-state bleach (vide infra), but the spectral dynamics identified in the data are nevertheless consistent with this general picture.

For the subtle changes associated with vibrational relaxation, single-wavelength measurements are better suited to determine the time scale of the process along the ${ }^{3}$ MLCT excited state. Plots of the kinetics measured at probe wavelengths on either side of the $405 \mathrm{~nm}$ isosbestic point for compound $\mathbf{1}$ are shown in Figure 5. There are processes occurring on multiple time scales: we will defer a discussion of the sub-picosecond dynamics and focus for now on kinetics associated with the spectral changes highlighted in Figure 4. The increase in signal amplitude to the blue side of $400 \mathrm{~nm}$ is clearly evident in

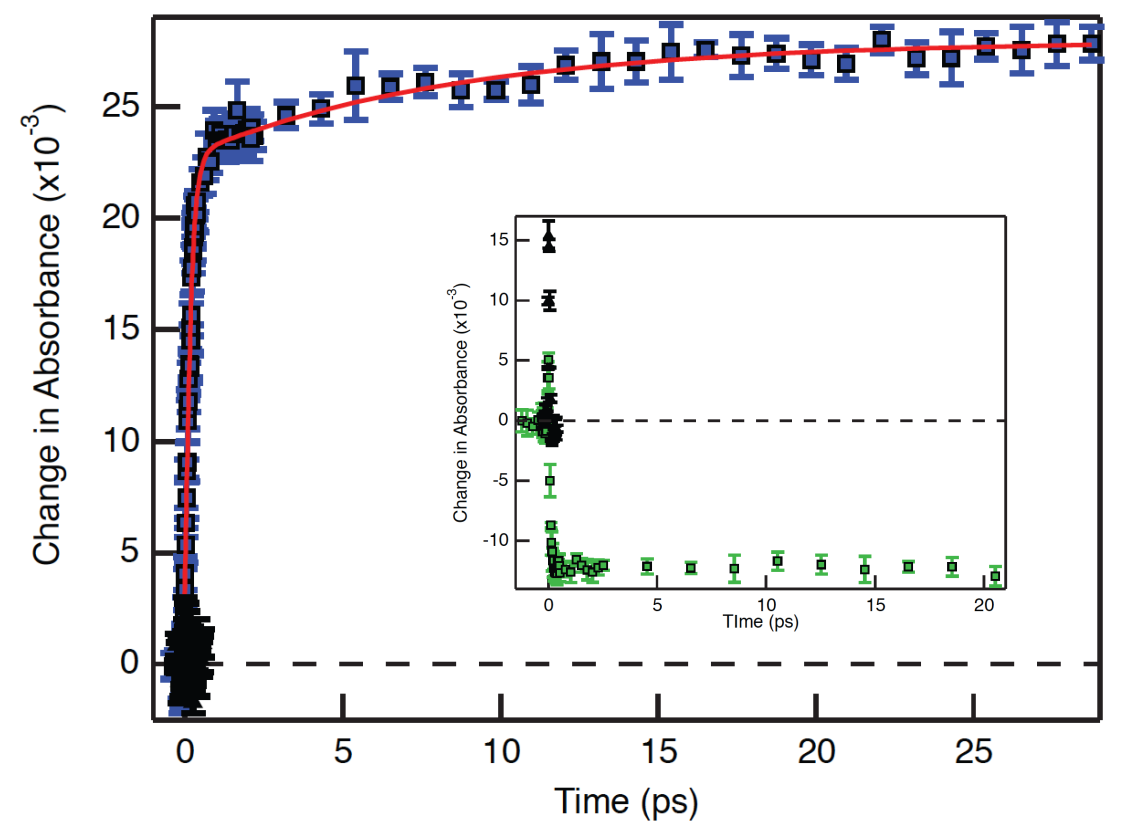

Figure 5. Single-wavelength kinetics for $\left[R u(b p y)_{2}(C N-M e-b p y)\right]^{2+}$ (1) at $390 \mathrm{~nm}$ following ${ }^{1} A_{1} \rightarrow{ }^{1} M L C T$ excitation at $490 \mathrm{~nm}$. The solvent response near $\Delta t=0$ is shown in black. The solid red line shows a fit of the kinetics to a biexponential kinetic model. The slower component corresponds to a time constant of $8 \pm$ 1 ps; an expanded view of the faster process can be found in the inset of Figure 7. Inset. Singlewavelength kinetics acquired at $\lambda_{\text {probe }}=430 \mathrm{~nm}$. See text for further details. 
Figure 5; these data can be described by an exponential with a time constant of $8 \pm 1$ ps that asymptotes to a baseline offset, i.e., the steady-state differential absorbance of the bpy radical anion associated with the fully thermalized ${ }^{3}$ MLCT excited state. This vibrational cooling time constant is in the same $5-20$ ps range that has been reported by us ${ }^{2,6,22-24,42}$ as well as a number of other researchers ${ }^{1,5,21,25,29,31,46,51,52}$ for this class of compounds.

It is interesting to note that data acquired at $430 \mathrm{~nm}$ (Figure 5, inset) do not show a corresponding kinetic feature for vibrational relaxation despite the qualitative connection mentioned above between these two portions of the differential spectra. Closer inspection of Figure $4 \mathrm{~b}$ reveals that, although the first two spectral traces trend in the manner described previously, there is no systematic modulation observed for $\lambda_{\text {probe }}>400 \mathrm{~nm}$. In fact, this situation is not uncommon (i.e., kinetics clearly in evidence in regions of net excited-state absorption not being observed in bleach regions) due to the fact that regions of net absorption are dominated by signals associated with the excited state whereas bleach regions predominantly reflect loss of ground-state absorption. Since the kinetics are associated with processes in the excited state, their manifestation can often be masked in regions where excited-state absorption represents a minority contribution to the overall signal.

Results similar to those just described for compound $\mathbf{1}$ were also obtained for complexes $\mathbf{2}$ and $\mathbf{3}$; analogous plots of the full spectrum and single-wavelength kinetics data for these two compounds are provided in Figures S3 - S6 of the Supplementary Materials.

3.2.2. Time-resolved Infrared Absorption Measurements. One of our primary goals in developing a $\mathrm{CN}$-substituted bipyridyl ligand was to provide an infrared tag that was spectrally well-isolated, coupled into the MLCT excited-state manifold, and readily identifiable in terms of its role in the excited state (e.g., either as a spectator ligand or housing the ligand-based electron of the MLCT state). The electronic asymmetry coupled with excitation energies specifically chosen to localize both the Franck-Condon and lowest-energy excited state onto the same ligand made it relatively straightforward to assign the $\sim 8 \mathrm{ps}$ process evident in Figure 6 to vibrational relaxation in the ${ }^{3}$ MLCT excited state. We can now explore the vibrational dynamics just inferred from electronic absorption spectroscopy in greater detail by directly examining the MLCT excited state in the region of the C-N stretch of the CN-Me-bpy ligand.

Figure 6 shows time-resolved infrared absorption data acquired on compound 1 following excitation at $490 \mathrm{~nm} .{ }^{53}$ As expected given the pump wavelength, a signal characteristic of the CN-Me-bpy-localized MLCT excited state is formed immediately upon excitation (left panel). Over the next several picoseconds, this absorption feature increases in intensity while it sharpens slightly, eventually yielding a spectral profile that matches what was observed using nanosecond time-resolved step-scan methods. ${ }^{32}$ The overall behavior is similar to what Browne, McGarvey and coworkers observed in their time-resolved 
(a)

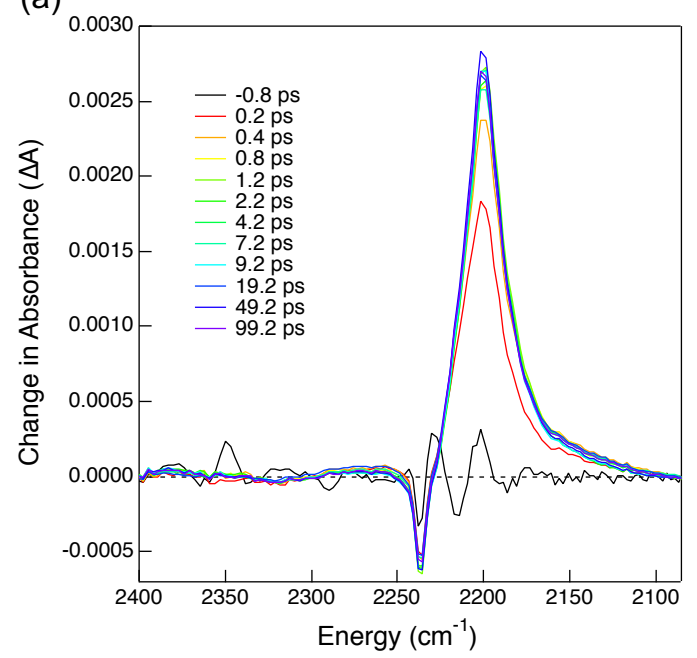

(b)

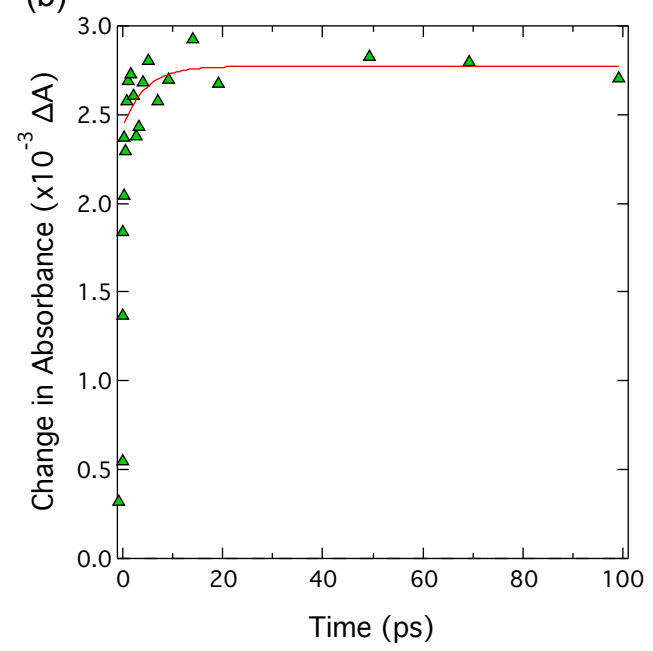

Figure 6. (a) Time resolved differential infrared absorption spectra for $\left[\mathrm{Ru}(\mathrm{bpy})_{2}(\mathrm{CN}-\mathrm{Me}-\mathrm{bpy})\right]^{2+}$ (1) collected in $\mathrm{CH}_{3} \mathrm{NO}_{2}$ solution following ${ }^{1} \mathrm{~A}_{1} \rightarrow{ }^{1} \mathrm{MLCT}$ excitation at $490 \mathrm{~nm}$. The inset numbers correspond to delay times for each spectrum. (b) Kinetic trace of the data plotted in part (a) sampled at the absorption maximum $\left(2200 \mathrm{~cm}^{-1}\right)$. The red line corresponds to a single exponential fit of the data, yielding a time constant of $3.0 \pm 1.5 \mathrm{ps}$.

infrared study of $\left[\mathrm{Ru}(\mathrm{bpy})_{3}\right]^{2+}:$ an increase in the intensity of the excited state absorption feature without any significant change in the energy or spectral profile of the band. ${ }^{55}$ The kinetics associated with this spectral evolution (as probed by the intensity of the band at its absorption maximum) are shown in the right-hand panel of Figure 6. It can be seen that the amplitude of the signal rises rapidly then levels off, with no further changes evident out to $\Delta \mathrm{t}=100 \mathrm{ps}$, the maximum delay to which we acquired data; obviously, this plateau corresponds to the signal for the fully thermalized, long-lived ${ }^{3}$ MLCT excited state which undergoes ground-state recovery on the microsecond time scale. ${ }^{32}$ The data can be fit to a single exponential kinetic model with a time constant of $3.0 \pm 1.5 \mathrm{ps}$, a value comparable to that reported for $\left[\mathrm{Ru}(\mathrm{bpy})_{3}\right]^{2+} \cdot{ }^{5}$

There are several aspects associated with the appearance of the transient IR spectra that are worth noting. First, the excited-state absorption band is somewhat broader and more Gaussian in shape (suggestive of inhomogeneous broadening) as compared to the ground-state absorption, which we have verified as Lorentzian based on an analysis of our previously published data FTIR data. ${ }^{32}$ This is reminiscent of what was observed in the nanosecond time-resolved infrared data and is likely a consequence of the near-degeneracy of the symmetric and asymmetric $\mathrm{CN}$ stretches associated with the bpy-CN ligand as well as solvation associated with the charge density on the ligand in the MLCT excited- 
state. The increase in the intensity of the CN stretch in the MLCT excited state relative to the ground state inferred by the disparate magnitudes of the transient absorption and ground-state bleach evident in Figure 6a prompted us to carry out infrared spectroelectrochemical measurements on compound $\mathbf{1}$. Indeed, these data reveal that the intensity of the $\mathrm{CN}$ stretch of the one-electron reduced form of $\left[\mathrm{Ru}(\mathrm{bpy})_{2}(\mathrm{CN}-\mathrm{Me}-\right.$ bpy) $]^{2+}$ is roughly an order of magnitude larger than the corresponding feature in the ground state. We suspect that the underlying reason for this is due to enhanced electronic-vibrational coupling between the cyano group and the $\pi$ system of the bipyridyl ligand that was highlighted in the TD-DFT calculations we reported previously, ${ }^{32}$ an interaction that could lead to an increase in the oscillator strength of the $\mathrm{CN}$ stretch in the MLCT excited state. A more detailed examination of this effect is currently underway, but these data nevertheless provide a qualitative explanation for the difference in the relative intensities of the two features seen in Figure 6a.

Perhaps more interesting is the fact that both the center peak position and bandwidth of the excitedstate absorption is virtually constant across most of the temporal window being sampled, suggesting that the $v(\mathrm{C} \equiv \mathrm{N}) \operatorname{mode}(\mathrm{s})$ are not engaged in significant anharmonic coupling to other low-frequency vibrations of the ligand framework on the time scale of these measurements. This allows us to come to a preliminary conclusion (one which will amplify in the next section) that any intramolecular vibrational redistribution dynamics occurring in the excited state are largely complete within the first picosecond following MLCT excitation. Analogous results with regard to both the spectral evolution of the (CN-Mebpy $^{-}$) signal and its associated kinetics were obtained for compounds $\mathbf{2}$ and $\mathbf{3}$ and are presented in Figures S7 and S8, respectively.

Although the time constants for vibrational cooling derived from the electronic and infrared absorption data are comparable ( $8 \pm 1$ ps and $3.0 \pm 1.5 \mathrm{ps}$, respectively), the slight discrepancy deserves comment. We suggest that this difference arises due to the nature of what is being probed in the two experiments. The data presented in Figure 6 corresponds to an examination of one specific vibrational mode of the molecule, namely the cyano group of the CN-Me-bpy ligand. While this moiety is strongly coupled into the MLCT excited state, it is a high-frequency mode that is also energetically isolated: because of this, the group is to a certain extent decoupled from most of the other vibrational modes of the compound. In addition, its high energy translates into a much faster vibrational period than lower frequency modes as well as imposing a limitation in terms of the amount of energy that it can assist in dissipating. The increase in IR intensity could be associated with the evolution of the ${ }^{3}$ MLCT electronic wavefunction in the course of vibrational relaxation, namely through an increase in conjugation between the $\mathrm{CN}$ groups and the rest of the ligand. Electronic absorption spectra, on the other hand, provide a window into the overall evolution of the exited state and are not mode-specific in this regard. 
Consequently, vibrational relaxation dynamics involving lower frequency modes - which are important for dissipating energy through intramolecular vibrational redistribution - can manifest in electronic spectra long after a higher-frequency vibration such as a cyano group has effectively cooled. This notion is indirectly supported by the work of Browne et al. on $\left[\mathrm{Ru}(\mathrm{bpy})_{3}\right]^{2+} .^{55}$ Their measurements and analysis keyed on the fingerprint region of the vibrational spectrum of that compound, which by its very nature provides a much broader snapshot of vibrational properties of the chromophore. The time constant these workers report is much closer to what we infer from electronic absorption spectroscopy. Although this is admittedly a minor point, it does appear that the incorporation of the $\mathrm{CN}$ group in our system has provided an unexpected glimpse into how different components of the molecule become involved in

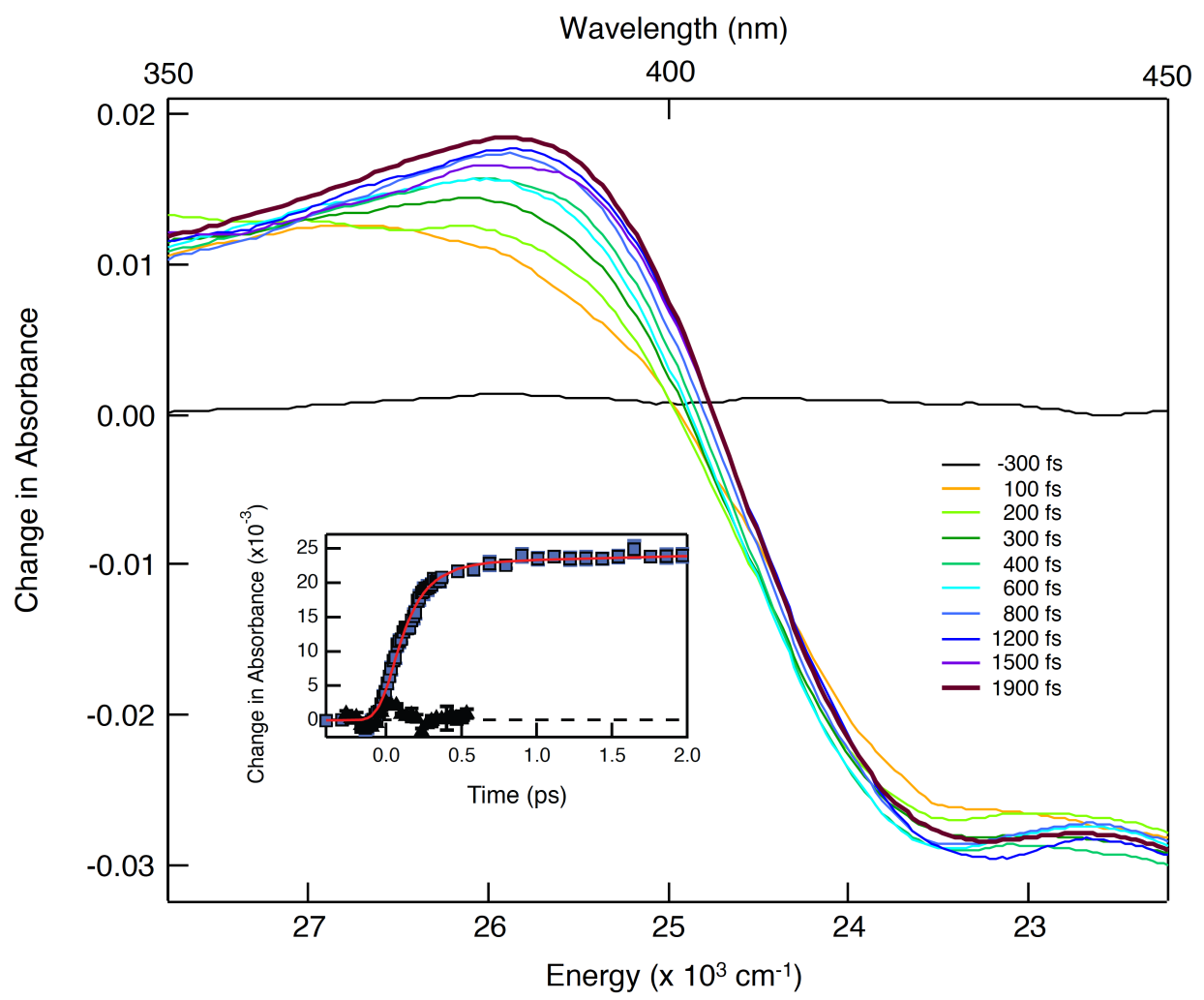

Figure 7. Differential electronic absorption spectra acquired for $\left[\mathrm{Ru}(b p y)_{2}(\mathrm{CN}-\mathrm{Me}-\mathrm{bpy})\right]^{2+}$ (1) in $\mathrm{CH}_{3} \mathrm{CN}$ solution over a delay window of $2 \mathrm{ps}$ following ${ }^{1} A_{1} \rightarrow{ }^{1} M L C T$ excitation at $490 \mathrm{~nm}$. The time delays corresponding to each spectral trace are indicated in the figure; the spectra have been corrected for chirp due to group velocity dispersion using global analysis and singular value decomposition. The inset figure is an expanded view of the data presented in Figure $5\left(\lambda_{\text {probe }}=390 \mathrm{~nm}\right)$ and tracks the increase in signal intensity evident in the spectral evolution. The time constant for the rise of the signal is $170 \pm 50 f_{S}$ and is assigned to intramolecular vibrational redistribution (IVR). See text for further details. 
energy dissipation. The main message, however, is that the results presented herein strongly support the notion that one can indeed obtain reliable information concerning vibrational relaxation dynamics from a careful analysis of transient electronic spectra.

3. 3. Sub-Picosecond Dynamics: Intramolecular Vibrational Energy Redistribution. In addition to the vibrational cooling identified by both time-resolved electronic and infrared absorption, there are additional dynamics evident in the evolution of the differential absorption spectra occurring on a subpicosecond time scale. In Figure 7 are plotted differential absorption spectra for compound $\mathbf{1}$ acquired at high temporal resolution over the first 2 picoseconds following MLCT excitation at $490 \mathrm{~nm}$. It can be seen that there is an increase in the intensity of the feature to the blue of the isosbestic points concomitant with a slight deepening of the bleach signal, suggestive of an overall sharpening of the transient absorption at $390 \mathrm{~nm}$. Qualitatively, the nature of the change in the spectral profile is very similar to what we described earlier for vibrational cooling (vide supra), but these changes are occurring on a significantly faster time scale. The inset of Figure 7 shows the kinetics associated with the rise of this signal at $390 \mathrm{~nm}$; this is essentially an expanded view of the first two picoseconds of the kinetic trace shown in Figure 6. The fit corresponds to a convolution of the instrument response function with a singleexponential amplitude rise reflecting the increase in signal intensity evident in the spectra near $390 \mathrm{~nm}$. The time constant derived from this fit is $170 \pm 50 \mathrm{fs}$, which we are attributing to intramolecular vibrational energy redistribution (IVR) based on the following considerations.

IVR refers to a process by which energy is redistributed amongst various vibrational degrees of freedom of the molecule that are not directly coupled to the electronic transition that was initially excited. There is no net energy loss to the surrounding environment associated with this process (e.g., no heat dissipation to the solvent), but the partitioning of energy from a small subset of vibrational modes to encompass a larger fraction of the vibrational manifold of the molecule, combined with evolution of the electronic wavefunction should influence the spectroscopic observables of the chromophore in some manner. The most straightforward probes of IVR are vibrational in nature; when direct spectral signatures are absent and/or difficult to discern, vibrational redistribution can manifest as subtle modulations upon an already formed positive or negative feature. Ultrafast time-resolved infrared absorption and timeresolved resonance Raman spectroscopies display very similar features that are frequently used to identify IVR, specifically the type of spectral shifting and bandshape evolution previously described in the context of vibrational cooling. ${ }^{52}$ In this model, the active vibrational modes contain all the excess energy imparted by the excitation pulse. As this energy is redistributed into other vibrational modes, the vibrational distribution broadens and modulation of the original spectral features can be observed. Because of the qualitatively similar imprint on excited-state absorption features, distinguishing between IVR and 

examine the $\mathrm{CO}$ and $\mathrm{C}=\mathrm{C}$ stretches of the ligand as a function of time. ${ }^{20}$ Both displayed a $300-700 \mathrm{fs}$ time constant; the typical blue-shift and band narrowing were observed for the $\mathrm{CO}$ stretch, whereas the $\mathrm{C}=\mathrm{C}$ lineshape was indicative of a non-Boltzmann distribution of thermal energy amongst vibrational modes. Given the data, IVR was assigned to the multi-hundred femtosecond time constant for both of these frequencies. In another relevant experiment, Hauser and coworkers studied the $\mathrm{C}-\mathrm{H}$ stretching and bending modes in the aromatic system of $\left[\mathrm{Ru}(\mathrm{bpy})_{3}\right]^{2+} \cdot{ }^{21}$ Upon $400 \mathrm{~nm}$ excitation, two bands centered at 1495 and $1545 \mathrm{~cm}^{-1}$ appeared, the second of which was initially broad but then narrowed within 2.8 ps. IVR was assigned in this case based primarily on the spectral features, time constant, and the amount of excess energy placed on the ligand with the near-UV excitation wavelength. And while visible pumpvisible probe experiments in which IVR is identified are rare, ${ }^{1,54}$ examples do exist that display the spectral modulations and time constants consistent with those reported for the more direct vibrationalprobe spectroscopies. It should be noted that the study of IVR dynamics as it occurs in transition metalcontaining systems is an area of growing interest. ${ }^{56-60}$

Kinetic processes detected by transient electronic absorption have typically been attributed to IVR on a comparative basis using previous reports on similar molecules. ${ }^{14,39}$ With the understanding of how signals associated with the IVR process manifest in complementary experiments, the signal from visible transient absorption can be rationalized. As seen in Figure 7, early time traces for complex 1 show an evolution of the shape of the transient absorption spectrum in both the bleach and absorption regions. Keeping in mind that changes in the appearance of a differential absorption spectrum reflects excited-state dynamics (i.e., the loss of ground-state absorption - the "bleach" - is static), the increase in intensity of the main absorption centered near $390 \mathrm{~nm}$ at the expense of absorbance on the high energy side of the band coupled with the increase in the magnitude of the bleach to the red of the isosbestic point indicates a sharpening of the underlying excited-state absorption. As mentioned previously, this is qualitatively similar to the signature for vibrational cooling because both processes - IVR and vibrational cooling - are depopulating higher energy vibrational levels of the excited-state potential energy surface. While there is an important fundamental difference between the two mechanisms - vibrational cooling dissipating energy to the surrounding medium versus IVR channeling it into other vibrational modes of the compound - the net effect of depopulating these higher energy vibrational quanta on the electronic absorption feature is the same.

Given the nature of the excited state (i.e., charge transfer), the observed spectral evolution could be 
associated with solvation dynamics. To address this possibility, we carried out analogous studies in two longer-chain nitrile solvents, namely $\mathrm{CH}_{3} \mathrm{CH}_{2} \mathrm{CN}$ and $\mathrm{CH}_{3}\left(\mathrm{CH}_{2}\right)_{2} \mathrm{CN}$. As we have reported previously, inertial solvent dynamics can couple to excited-state evolution in this class of complexes, and although the time scale of what we are assigning to IVR is significantly longer than the $\sim 60 \mathrm{fs}$ inertial response of $\mathrm{CH}_{3} \mathrm{CN}$, we wanted to verify that the process we are observing was intramolecular in nature. Indeed, the data acquired in all three solvents were indistinguishable from each other, thereby providing additional support for our conclusion.

Although the results just described point to an intramolecular origin of the dynamics reflected in Figure 7, it is important to note that, on these time scales, the evolution of the differential electronic absorption spectrum is also expected to be influenced by changes in the composition and spatial characteristics of the electronic wavefunction as the system evolves from the Franck-Condon to lowestenergy excited state of the system. Parsing out the relative contributions from these possible contributions should be possible by carrying out systematic studies as a function of excitation wavelength and perhaps even temperature. This latter point notwithstanding, we believe that the data in Figure 7 are consistent both qualitatively and quantitatively with what one would expect for an IVR signature in differential electronic absorption data in this class of compounds.

Corresponding data were also acquired on complexes $\mathbf{2}$ and $\mathbf{3}$ and are provided in Figure S9 and S10, respectively. Qualitatively, we see similar features in terms of spectral evolution in these two complexes as we have just described for complex $\mathbf{1}$ but the magnitude of the changes are systematically attenuated as bpy ligands are replaced by CN-Me-bpy. Indeed, the data on complex 3 shows almost no discernable dynamics on a sub-200 fs time scale. In contrast to complex 1, in which the excited state can only reside on a single ligand, the dynamics associated with complexes $\mathbf{2}$ and $\mathbf{3}$ are potentially more complex due to the fact that the spatial localization of the initially formed excited state is not as clearly defined. We have previously discussed the role of solvent in driving excited-state charge localization, ${ }^{39}$ reflecting a balance between the strength of interligand coupling and the energetic asymmetry of the environment for systems involving higher degrees of degeneracy. Indeed, the interplay of evolving excited-state delocalization, inertial solvent response, and vibrational relaxation dynamics is an extremely interesting topic, but a detailed analysis along these lines is beyond the scope of this report.

3.4. High Energy Excitation. From the orbital pictures in Figure 2, it is clear that excitation of 1 at higher energy (i.e. $400 \mathrm{~nm}$ ) results in a different Franck-Condon state than the low energy $490 \mathrm{~nm}$ excitation, one that is localized on the unsubstituted bpy ligand(s). Changing the excitation wavelength of complex 1 from $490 \mathrm{~nm}$ to $400 \mathrm{~nm}$ should therefore result in additional excited-state dynamics pathways, most notably interligand electron transfer (ILET) to the CN-Me-bpy ligand. As a preliminary 
(a)

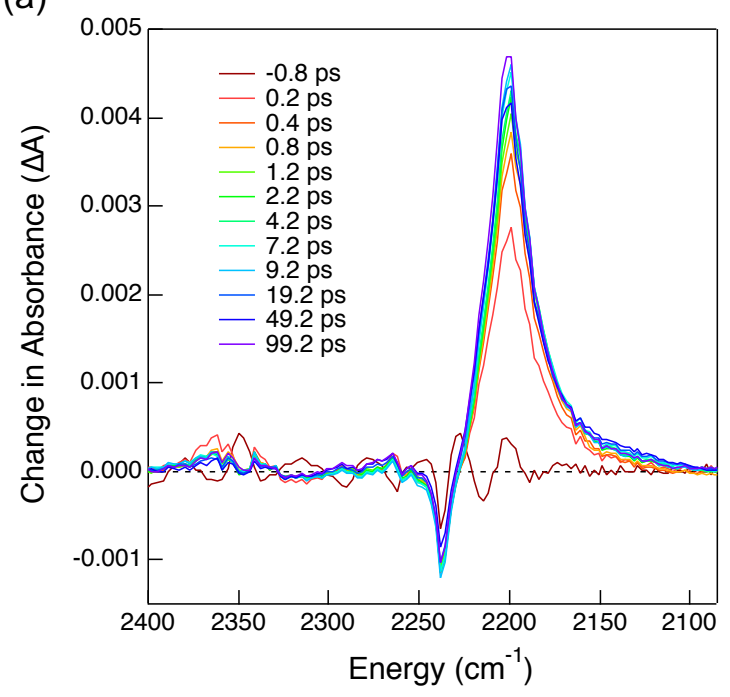

(b)

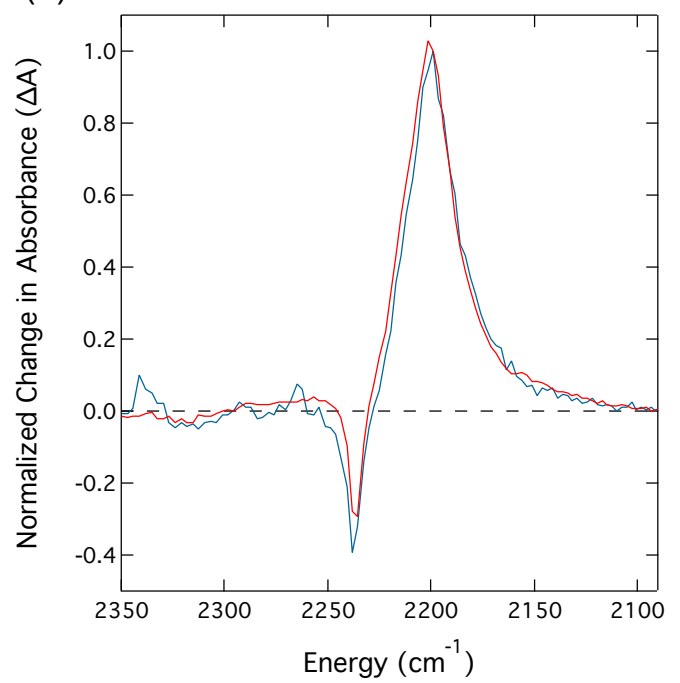

Figure 8. (a) Time-resolved differential infrared absorption spectra for $\left[\mathrm{Ru}(\mathrm{bpy})_{2}(\mathrm{CN}-\mathrm{Me}-\mathrm{bpy})\right]^{2+}$ (1) collected in $\mathrm{CH}_{3} \mathrm{NO}_{2}$ solution following ${ }^{1} A_{1} \rightarrow{ }^{1} \mathrm{MLCT}$ excitation at $400 \mathrm{~nm}$. The inset numbers correspond to delay times for each spectrum. (b) Comparison of time-resolved differential infrared spectra for $\left[\mathrm{Ru}(\mathrm{bpy})_{2}(\mathrm{CN}-\mathrm{Me} \text {-bpy) }]^{2+}\right.$ (1) at a time delay of $1 \mathrm{ps}$ following excitation at $400 \mathrm{~nm}$ (blue) and $490 \mathrm{~nm}(\mathrm{red})$. The fact that these spectra superimpose indicates that processes associated with forming the lowest-energy ${ }^{3}$ MLCT excited state (e.g., interligand electron transfer) are complete in $<1 \mathrm{ps.}$ 
high-energy excitation of complexes $\mathbf{1}$ and $\mathbf{2}$, this observation is consistent with the notion that interligand electron transfer is occurring on a sub-picosecond time scale in these compounds. It is also possible that the angle between the electronic and vibrational transition dipole moment vectors is accidentally close to the magic angle, the anisotropy is negligible from the very beginning and its time dependence thus provides no information on the excited-state dynamics. A similar situation would arise of the optical laser pulse excites several electronic transitions with different transition moment directions. A detailed investigation into the excited-state dynamics associated with high-energy excitation of this system including time-resolved polarized electronic and infrared spectroscopies - is currently underway and will be the subject of a future report.

\section{Concluding Comments}

The time-resolved infrared and visible absorption experiments presented herein comprise a selfconsistent picture of vibrational relaxation dynamics in a $\mathrm{Ru}(\mathrm{II})$ polypyridyl charge transfer system. The utility of the chemical platform that we have developed lies in the incorporation of a functional group that is coupled into the charge-transfer manifold of the chromophore while at the same time possessing vibrational signatures in a relatively isolated region of the infrared spectrum. This provided a unique opportunity to simultaneously probe electronic and nuclear dynamics associated with charge transfer-state evolution. Vibrational cooling - the process by which energy is dissipated into the surrounding environment - occurs on 1-10 ps timescale in all three of the complexes studied. This conclusion was reached through indirect means through an analysis of changes in the differential time-resolved electronic absorption data, then corroborated through direct, time-resolved infrared absorption measurements using the $\mathrm{CN}$ moiety on the bipyridyl ligand. This timescale has been observed in other ruthenium bipyridyl systems, most definitively in the work of Browne, McGarvey and coworkers through their time-resolved resonance Raman measurements of $\left[\mathrm{Ru}(\mathrm{bpy})_{3}\right]^{2+} \cdot{ }^{55}$ Qualitatively similar spectral changes were also observed in the electronic absorption data but on a much faster time scale. Analysis of these data showed that these features are consistent with expectations for intramolecular vibrational energy redistribution following photoexcitation occurring with a time constant of $170 \pm 50 \mathrm{fs}$.

It should be noted that the applicability of the methodology we have employed is not limited to complexes of $\mathrm{Ru}(\mathrm{II})$. Indeed, studies are currently underway to exploit this combined spectroscopic and computational approach to compounds of first-row transition metals (e.g., Fe(II)) in order to probe the interplay between electronic and vibrational degrees of freedom under conditions where kinetic competition among these processes is of paramount importance for understanding (and ultimately controlling) the photophysical and photochemical properties of such compounds. 
Acknowledgments: The authors would like to thank Dr. John A. Kouzelos for his assistance with DFT calculations, and Jennifer N. Miller for data collection in other solvents. This work was supported by the Chemical Sciences, Geosciences, and Biosciences Division, Office of Basic Energy Sciences, Office of Science, U.S. Department of Energy through grant no. DE-FG02-01ER15282 (JKM) and STFC (United Kingdom) and the Czech Science Foundation Grant 17-011375 (AV).

Supporting Information Available: Complete citation for reference 34, comparison of nanosecond and picosecond transient absorption spectra for complexes $\mathbf{2}$ and $\mathbf{3}$, and data for complex $\mathbf{2}$. This material is available free of charge via the Internet at http://pubs.acs.org. 


\section{References}

(1) Peteanu, L. A.; Schoenlein, R. W.; Wang, Q.; Mathies, R. A.; Shank, C. V. The First Step in Vision Occurs in Femtoseconds: Complete Blue and Red Spectral Studies. Proc. Natl. Acad. Sci. U.S.A. 1993, 90, 11762.

(2) Kuciauskas, D.; Monat, J. E.; Villahermosa, R.; Gray, H. B.; Lewis, N. S.; McCusker, J. K. Transient Absorption Spectroscopy of Ruthenium and Osmium Polypyridyl Complexes Adsorbed onto Nanocrystalline $\mathrm{TiO}_{2}$ Photoelectrodes. J. Phys. Chem. B 2002, 106, 9347-9358.

(3) Hoffman, D. P.; Ellis, S. R.; Mathies, R. A. Characterization of a Conical Intersection in a ChargeTransfer Dimer with Two-Dimensional Time-Resolved Stimulated Raman Spectroscopy. J. Phys. Chem. A 2014, 118, 4955-4965.

(4) Hauser, A.; Reber, C. Spectroscopy and Chemical Bonding in Transition Metal Complexes. Struct. Bonding (Berlin, Ger.) 2016, 172, 291-312.

(5) Frontiera, R. R.; Dasgupta, J.; Mathies, R. A. Probing Interfacial Electron Transfer in Coumarin 343 Sensitized $\mathrm{TiO}_{2}$ Nanoparticles with Femtosecond Stimulated Raman. J. Am. Chem. Soc. 2009, $131,15630-15632$.

(6) Záliš, S.; Busby, M.; Kotrba, T.; Matousek, P.; Towrie, M.; Vlček, A., Jr. Excited-State Characters and Dynamics of $\left[\mathrm{W}(\mathrm{CO})_{5}-(4\right.$-cyanopyridine $\left.)\right]$ and $\left[\mathrm{W}(\mathrm{CO})_{5}\right.$ (piperidine)] Studied by Picosecond Time-Resolved IR and Resonance Raman Spectroscopy and DFT Calculations: Roles of W $\rightarrow \mathrm{L}$ and $\mathrm{W} \rightarrow \mathrm{CO}$ MLCT and LF Excited States Revised. Inorg. Chem. 2004, 43, 1723-1734.

(7) Hedley, G. J.; Ruseckas, A.; Samuel, I. D. Ultrafast Intersystem Crossing in a Red Phosphorescent Iridium Complex. J. Phys. Chem. A 2009, 113, 2-4.

(8) Marin, T. W.; Homoelle, B. J.; Spears, K. G. Ultrafast Electron Transfer in the $\left[\mathrm{Co}(\mathrm{Cp})_{2} \mid \mathrm{V}(\mathrm{CO})_{6}\right]$ Radical Pair. J. Phys. Chem. A 2002, 106, 1152-1166.

(9) Blanco-Rodríguez, A. M.; Busby, M.; Ronayne, K.; Towrie, M.; Grădinaru, C.; Sudhamsu, J.; Sýkora, J.; Hof, M.; Zálišs, S.; Di Bilio, A. J.; Crane, B. R.; Gray, H. B.; Vlček, A., Jr. Relaxation Dynamics of Pseudomonas aeruginosa $\operatorname{Re}^{\mathrm{I}}(\mathrm{CO})_{3}(\alpha$-diimine $)(\mathrm{His} \mathrm{X})^{+}(\mathrm{X}=83,107,109,124$, 126)Cu ${ }^{\text {II }}$ Azurins. J. Am. Chem. Soc. 2009, 131, 11788-11800.

(10) Baskin, J. S.; Yu, H.-Z.; Zewail, A. H. Ultrafast Dynamics of Porphyrins in the Condensed Phase: I. Free Base Tetraphenylporphyrin. J. Phys. Chem. A 2002, 106, 9837-9844.

(11) Ernsting, N. P.; Kovalenko, S. A.; Senyushkina, T.; Saam, J.; Farztdinov, V. Wave-Packet-Assisted 
(12) Hedley, G. J.; Ruseckas, A.; Samuel, I. D. W. Vibrational Energy Flow Controls Internal Conversion in a Transition Metal Complex. J. Phys. Chem. A 2010, 114, 8961-8968.

(13) Delor, M.; Keane, T.; Scattergood, P. A.; Sazanovich, I. V.; Greetham, G. M.; Towrie, M.; Meijer, A. J. H. M.; Weinstein, J. A. On the Mechanism of Vibrational Control of Light-Induced Charge Transfer in Donor-Bridge-Acceptor Assemblies. Nat. Chem. 2015, 7, 689-695.

(14) Damrauer, N. H.; Cerullo, G.; Yeh, A.; Boussie, T. R.; Shank, C. V.; McCusker, J. K. Femtosecond Dynamics of Excited-State Evolution in $\left[\mathrm{Ru}(\mathrm{bpy})_{3}\right]^{2+}$. Science 1997, 275, 54-57.

(15) Hedley, G. J.; Ruseckas, A.; Liu, Z.; Lo, S. C.; Burn, P. L.; Samuel, I. D. W. Iridium Metal Complexes as an Unambiguous Probe of Intramolecular Vibrational Redistribution. J. Am. Chem. Soc. 2008, 130, 11842-11843.

(16) Hedley, G. J.; Ruseckas, A.; Samuel, I. D. W. Ultrafast Luminescence in $\operatorname{Ir}(\text { ppy })_{3}$. Chem. Phys. Lett. 2008, 450, 292-296.

(17) Mizutani, Y.; Uesugi, Y.; Kitagawa, T. Intramolecular Vibrational Energy Redistribution and Intermolecular Energy Transfer in the (d,d) Excited State of Nickel Octaethylporphyrin. J. Chem. Phys. 1999, 111, 8950-8962.

(18) Mizutani, Y.; Kitagawa, T. Mode Dependence of Vibrational Energy Redistribution in Nickel Tetraphenylporphyrin Probed by Picosecond Time-Resolved Resonance Raman Spectroscopy: Slow IVR to Phenyl Peripherals. Bull. Chem. Soc. Jpn. 2002, 75, 965-971.

(19) Banno, M.; Iwata, K.; Hamaguchi, H. O. Intra- and Intermolecular Vibrational Energy Transfer in Tungsten Carbonyl Complexes $\mathrm{W}(\mathrm{CO})_{5}(\mathrm{X})\left(\mathrm{X}=\mathrm{CO}, \mathrm{CS}, \mathrm{CH}_{3} \mathrm{CN}\right.$, and $\left.\mathrm{CD}_{3} \mathrm{CN}\right)$. J. Chem. Phys. 2007, 126, 204501

(20) Maçôas, E. M. S.; Mustalahti, S.; Myllyperkiö, P.; Kunttu, H.; Pettersson, M. Role of Vibrational Dynamics in Electronic Relaxation of $\mathrm{Cr}(\mathrm{acac})_{3}$. J. Phys. Chem. A 2015, 119, 2727-2734.

(21) Sun, Q.; Dereka, B.; Vauthey, E.; Daku, L. M. L.; Hauser, A. Ultrafast Transient IR Spectroscopy and DFT Calculations of Ruthenium(II) Polypyridyl Complexes. Chem. Sci. 2017, 8, 223-230.

(22) Damrauer, N. H.; McCusker, J. K. Ultrafast Dynamics in the Metal-to-Ligand Charge Transfer Excited-State Evolution of $\left[\mathrm{Ru}\left(4,4^{\prime} \text {-diphenyl-2,2'-bipyridine }\right)_{3}\right]^{2+}$. J. Phys. Chem. A 1999, 103, $8440-8446$. 
(23) Juban, E. A.; McCusker, J. K. Ultrafast Dynamics of ${ }^{2}$ E State Formation in $\mathrm{Cr}(\mathrm{acac})_{3} . J$. Am. Chem. Soc. 2005, 127, 6857-6865.

(24) Schrauben, J. N.; Dillman, K. L.; Beck, W. F.; McCusker, J. K. Vibrational Coherence in the Excited State Dynamics of $\mathrm{Cr}(\mathrm{acac})_{3}$ : Probing the Reaction Coordinate for Ultrafast Intersystem Crossing. Chem. Sci. 2010, 1, 405-410.

(25) King, J. C.; Zhang, J. Z.; Schwartz, B. J.; Harris, C. B. Vibrational Relaxation of $\mathrm{M}(\mathrm{CO})_{6}(\mathrm{M}=\mathrm{Cr}$, Mo, W): Effect of Metal Mass on Vibrational Cooling Dynamics and Non-Boltzmann Internal Energy Distributions. J. Chem. Phys. 1993, 99, 7595-7601.

(26) Simon, J. D.; Xie, X. L. Photodissociation of $\mathrm{Cr}(\mathrm{CO})_{6}$ in 1-Propanol and 2-Propanol - Effect of Solvent Structure on the Mechanisms of Formation of $\mathrm{Cr}(\mathrm{CO})_{5}(\mathrm{OHR})$ from Photogenerated $\mathrm{Cr}(\mathrm{CO})_{5}(\mathrm{ROH})$. J. Phys. Chem. 1989, 93, 291-293.

(27) Xie, X. L.; Simon, J. D. Picosecond Time-Resolved Absorption Studies of the Solvation of Cr(CO) 5 in Alcohols: A Unimolecular Kinetic Model for the Formation of $\mathrm{Cr}(\mathrm{CO})_{5}(\mathrm{OHR})$ from Photogenerated $\mathrm{Cr}(\mathrm{CO})_{5}(\mathrm{ROH}) . J$. Am. Chem. Soc. 1990, 112, 1130-1136.

(28) Tominaga, K.; Kliner, D. A. V.; Johnson, A. E.; Levinger, N. E.; Barbara, P. F. Femtosecond Experiments and Absolute Rate Calculations on Intervalence Electron Transfer of Mixed-Valence Compounds. J. Chem. Phys. 1993, 98, 1228-1243.

(29) Maçôas, E. M.; Kananavicius, R.; Myllyperkiö, P.; Pettersson, M.; Kunttu, H. Ultrafast Electronic and Vibrational Energy Relaxation of $\mathrm{Fe}(\text { acetylacetonate) })_{3}$ in Solution. J. Phys. Chem. A 2007, 111, 2054-2061.

(30) Yoon, S.; Kukura, P.; Stuart, C. M.; Mathies, R. A. Direct Observation of the Ultrafast Intersystem Crossing in Tris(2,2'-bipyridine)ruthenium(II) Using Femtosecond Stimulated Raman Spectroscopy. Mol. Phys. 2006, 104, 1275-1282.

(31) Henry, W.; Coates, C. G.; Brady, C.; Ronayne, K. L.; Matousek, P.; Towrie, M.; Botchway, S. W.; Parker, A. W.; Vos, J. G.; Browne, W. R.; McGarvey, J. J. The Early Picosecond Photophysics of Ru(II) Polypyridyl Complexes: A Tale of Two Timescales. J. Phys. Chem. A 2008, 112, 4537-4544.

(32) McCusker, C. E.; McCusker, J. K. Synthesis and Spectroscopic Characterization of CN-Substituted Bipyridyl Complexes of Ru(II). Inorg. Chem. 2011, 50, 1656-1669.

(33) Greetham, G. M.; Burgos, P.; Cao, Q.; Clark, I. P.; Codd, P. S.; Farrow, R. C.; George, M. W.; Kogimtzis, M.; Matousek, P.; Parker, A. W. et al. ULTRA: A Unique Instrument for TimeResolved Spectroscopy. Appl. Spec. 2010, 64, 1311-1319. 
(34) Frisch, M. J.; Trucks, G. W.; Schlegel, H. B.; Scuseria, G. E.; Robb, M. A.; Cheeseman, J. R.; Montgomery, J., J. A.; Vreven, T.; Kudin, K. N.; Burant, J. C.; et. al. Gaussian 03, revision D.01; Gaussian, Inc., Wallingford CT, 2004.

(35) Monat, J. E.; Rodriguez, J. H.; McCusker, J. K. Ground- and Excited-State Electronic Structures of the Solar Cell Sensitizer Bis(4,4'-dicarboxylato-2,2'-bipyridine)bis(isothiocyanato)ruthenium(II). $J$. Phys. Chem. A 2002, 106, 7399-7406.

(36) Wawire, C. M.; Jouvenot, D.; Loiseau, F.; Baudin, P.; Liatard, S.; Njenga, L.; Kamau, G. N.; Casida, M. E. Density-Functional Study of Luminescence in Polypyridine Ruthenium Complexes. J. Photochem Photobiol. A 2014, 276, 8-15.

(37) Tomasi, J.; Mennucci, B.; Cammi, R. Quantum Mechanical Continuum Solvation Models. Chem. Rev. 2005, 105, 2999-3093.

(38) Yeh, A. T.; Shank, C. V.; McCusker, J. K. Ultrafast Electron Localization Dynamics Following Photo-Induced Charge Transfer. Science 2000, 289, 935-938.

(39) Mabrouk, P. A.; Wrighton, M. S. Resonance Raman Spectroscopy of the Lowest Excited State of Derivatives of Tris(2,2'-bipyridine)ruthenium(II): Substituent Effects on Electron Localization in Mixed-Ligand Complexes. Inorg. Chem. 1986, 25, 526-531.

(40) Howell, S. L.; Gordon, K. C.; Waterland, M. R.; Leung, K. H.; Phillips, D. L. Resonance Raman Excitation Profile of a Ruthenium(II) Complex of Dipyrido[2,3-a:3',2'-c]phenazine. J. Phys. Chem. A 2006, 110, 11194-11199.

(41) Fantacci, S.; Ronca, E.; De Angelis, F. Impact of Spin-Orbit Coupling on Photocurrent Generation in Ruthenium Dye-Sensitized Solar Cells. J. Phys. Chem. Lett. 2014, 5, 375-380.

(42) Liard, D. J.; Busby, M.; Matousek, P.; Towrie, M.; Vlček, A., Jr. Picosecond Relaxation of ${ }^{3}$ MLCT Excited states of $\left[\operatorname{Re}(\operatorname{Etpy})(\mathrm{CO})_{3}(\mathrm{dmb})\right]^{+}$and $\left[\operatorname{Re}(\mathrm{Cl})(\mathrm{CO})_{3}(\mathrm{bpy})\right]$ as Revealed by Time-Resolved Resonance Raman, UV-vis, and IR Absorption Spectroscopy. J. Phys. Chem. A 2004, 108, 23632369.

(43) McCamant, D. W.; Kukura, P.; Mathies, R. A. Femtosecond Time-Resolved Stimulated Raman Spectroscopy: Application to the Ultrafast Internal Conversion in $\beta$-Carotene. J. Phys. Chem. A 2003, 107, 8208-8214.

(44) Asbury, J. B.; Wang, Y.; Lian, T. Time-Dependent Vibration Stokes Shift During Solvation: Experiment and Theory. Bull. Chem. Soc. Jpn. 2002, 75, 973-983. 
(45) El Nahhas, A.; Consani, C.; Blanco-Rodríguez, A. M.; Lancaster, K. M.; Braem, O.; Cannizzo, A.; Towrie, M.; Clark, I. P.; Záliš, S.; Chergui, M.; Vlček, A., Jr. Ultrafast Excited-State Dynamics of Rhenium(I) Photosensitizers $\left[\operatorname{Re}(\mathrm{Cl})(\mathrm{CO})_{3}(\mathrm{~N}, \mathrm{~N})\right]$ and $\left[\operatorname{Re}(\text { imidazole })(\mathrm{CO})_{3}(\mathrm{~N}, \mathrm{~N})\right]^{+}:$Diimine Effects. Inorg. Chem. 2011, 50, 2932-2943.

(46) Maçôas, E. M.; Kananavicius, R.; Myllyperkiö, P.; Pettersson, M.; Kunttu, H. Relaxation Dynamics of $\mathrm{Cr}(\mathrm{acac})_{3}$ Probed by Ultrafast Infrared Spectroscopy. J. Am. Chem. Soc. 2007, 129, 8934-8935.

(47) While these data cannot discriminate for the spin of the excited state - both ${ }^{1}$ MLCT and ${ }^{3} \mathrm{MLCT}$ manifolds will have spin-allowed absorption features and therefore cannot be easily distinguished using electronic absorption spectroscopy a priori - the work of Okada ${ }^{60}$ and Chergui ${ }^{61}$ in particular indicate a sub-50 fs time scale for intersystem crossing in $\left[\mathrm{Ru}(\mathrm{bpy})_{3}\right]^{2+}$. It is therefore likely that these data correspond to absorptions of the ${ }^{3}$ MLCT state of compound $\mathbf{1}$.

(48) Lytle, F. E.; Hercules, D. M. The Luminescence of Tris(2,2'-bipyridine)ruthenium(II) Dichloride. J. Am. Chem. Soc. 1969, 91, 253-257.

(49) Wallin, S.; Davidsson, J.; Modin, J.; Hammarström, L. Femtosecond Transient Absorption Anisotropy Study on $\left[\mathrm{Ru}(\mathrm{bpy})_{3}\right]^{2+}$ and $\left[\mathrm{Ru}(\mathrm{bpy})(\mathrm{bpy})_{4}\right]^{2+}$. Ultrafast Interligand Randomization of the MLCT State. J. Phys. Chem. A 2005, 109, 4697-4704.

(50) El Nahhas, A.; Cannizzo, A.; van Mourik, F.; Blanco-Rodríguez, A. M.; Záliš, S.; Vlček, A., Jr.; Chergui, M. Ultrafast Excited-State Dynamics of $\left[\operatorname{Re}(\mathrm{L})(\mathrm{CO})_{3}(\mathrm{bpy})\right]^{\mathrm{n}}$ Complexes: Involvement of the Solvent. J. Phys. Chem. A 2010, 114, 6361-6369.

(51) Shaw, G. B.; Styers-Barnett, D. J.; Gannon, E. Z.; Granger, J. C.; Papanikolas, J. M. Interligand Electron Transfer Dynamics in $\left[\mathrm{Os}(\mathrm{bpy})_{3}\right]^{2+}$ : Exploring the Excited State Potential Surfaces with Femtosecond Spectroscopy. J. Phys. Chem. A 2004, 108, 4998-5006.

(52) Hamm, P.; Ohline, S. M.; Zinth, W. Vibrational Cooling After Ultrafast Photoisomerization of Azobenzene Measured by Femtosecond Infrared Spectroscopy. J. Chem. Phys. 1997, 106, 519-529.

(53) Due to potential interference from the nitrile stretch of $\mathrm{CH}_{3} \mathrm{CN}$, these data were acquired in $\mathrm{CH}_{3} \mathrm{NO}_{2}$ solution. Our initial report on this class of chromophores ${ }^{32}$ demonstrated that the photophysical properties of this system are essentially identical in these two solvents.

(54) Rafiq, S.; Scholes, G. D. Slow Intramolecular Vibrational Relaxation Leads to Long-Lived ExcitedState Wavepackets. J. Phys. Chem. A 2016, 120, 6792-6799. 
(55) Browne, W. R.; McGarvey, J. J. The Raman Effect and Its Application to Electronic Spectroscopies in Metal-Centered Species: Techniques and Investigations in Ground and Excited States. Coord. Chem. Rev. 2007, 251, 454-473.

(56) Abraham, B.; Fan, H.; Galoppini, E.; Gundlach, L. Vibrational Spectroscopy on Photoexcited DyeSensitized Films via Pump-Defenerate Four-Wave Mixing. J. Phys. Chem. A 2018, 122, 2039-2045.

(57) Jiang, Y.; Liu, L.C.; Muller-Werkmeister, H.M.; Lu, C.; Zhang, D.; Field, R.L.; Sarracini, A.; Moriena, G.; Collet, E.; Miller, R.J.D. Structural Dynamics upon Photoexcitation in a Spin Crossover Crystal Probes with Femtosecond Electron Diffraction. Angew. Chem.Int. Ed. 2017, 56, $7130-7134$.

(58) Borgwardt, M.; Wilke, M.; Kiyan, I.Y.; Aziz, E.F. Ultrafast excited states dynamics of $\left.[\text { Ru(bpy })_{3}\right]^{2+}$ dissolved in ionic liquids. Phys. Chem. Chem. Phys. 2018, 18, 28893-28900.

(59) Fan, Y.; Dong, X.; Feng, M.; Zhao, J.; Wang, J. Central-metal effect on intramolecular vibrational energy transfer of $\mathrm{M}(\mathrm{CO})_{5} \mathrm{Br}(\mathrm{M}=\mathrm{Mn}, \mathrm{Re})$ probed by two-dimensional infrared spectroscopy. Phys. Chem. Chem. Phys. 2018, 20, 3637-3647.

(60) Eckert, P.A.; Kubarych, K.J. Oxidation-state Dependent Vibrational Dynamics Probed with 2D-IR. J. Phys. Chem. A 2017, 121, 2896-2902.

(61) Önfelt, B.; Lincoln, P.; Nordén, B.; Baskin, J. S.; Zewail, A. H. Femtosecond Linear Dichroism of DNA-Intercalating Chromophores: Solvation and Charge Separation Dynamics of $\left[\mathrm{Ru}(\text { phen })_{2} \mathrm{dppz}\right]^{2+}$ Systems. Proc. Natl. Acad. Sci. U.S.A. 2000, 97, 5708-5713.

(62) Stark, C. W.; Schreier, W. J.; Lucon, J.; Edwards, E.; Douglas, T.; Kohler, B. Interligand Electron Transfer in Heteroleptic Ruthenium(II) Complexes Occurs on Multiple Time Scales. J. Phys. Chem. A 2015, 119, 4813-4824.

(63) Hoff, D. A.; Silva, R.; Rego, L. G. C. Subpicosecond Dynamics of Metal-to-Ligand ChargeTransfer Excited states in Solvated $\left[\mathrm{Ru}(\mathrm{bpy})_{3}\right]^{2+}$ Complexes. J. Phys. Chem. C 2011, 115, 1561715626.

(64) Although we did not collect data out to a sufficiently long time delay to confirm for this particular system, complete depolarization of all transient signals due to rotational correlation effects are expected to occur on a time scale of many tens of picoseconds for this class of compounds in solvents with viscosities similar to that of $\mathrm{CH}_{3} \mathrm{CN}$ and $\mathrm{CH}_{3} \mathrm{NO}_{2}$. 


\section{TOC Graphic}

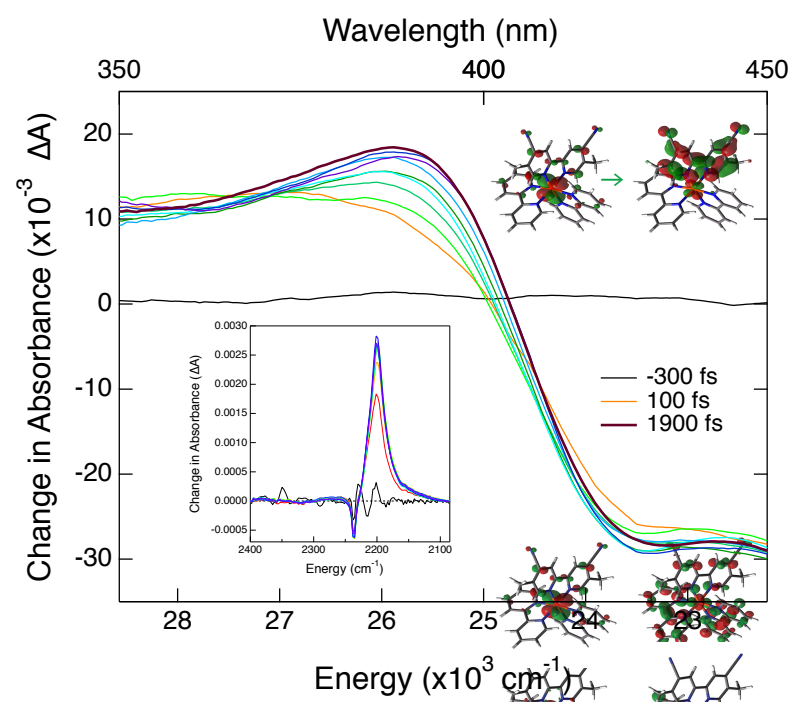

